\title{
Laboratory Experimentation of Guidance and Control of Spacecraft During On-orbit Proximity Maneuvers
}

\author{
Jason S. Hall and Marcello Romano \\ Naval Postgraduate School \\ Monterey, CA, USA
}

\section{Introduction}

The traditional spacecraft system is a monolithic structure with a single mission focused design and lengthy production and qualification schedules coupled with enormous cost. Additionally, there rarely, if ever, is any designed preventive maintenance plan or re-fueling capability. There has been much research in recent years into alternative options. One alternative option involves autonomous on-orbit servicing of current or future monolithic spacecraft systems. The U.S. Department of Defense (DoD) embarked on a highly successful venture to prove out such a concept with the Defense Advanced Research Projects Agency's (DARPA's) Orbital Express program. Orbital Express demonstrated all of the enabling technologies required for autonomous on-orbit servicing to include refueling, component transfer, autonomous satellite grappling and berthing, rendezvous, inspection, proximity operations, docking and undocking, and autonomous fault recognition and anomaly handling (Kennedy, 2008). Another potential option involves a paradigm shift from the monolithic spacecraft system to one involving multiple interacting spacecraft that can autonomously assemble and reconfigure. Numerous benefits are associated with autonomous spacecraft assemblies, ranging from a removal of significant intra-modular reliance that provides for parallel design, fabrication, assembly and validation processes to the inherent smaller nature of fractionated systems which allows for each module to be placed into orbit separately on more affordable launch platforms (Mathieu, 2005).

With respect specifically to the validation process, the significantly reduced dimensions and mass of aggregated spacecraft when compared to the traditional monolithic spacecraft allow for not only component but even full-scale on-the-ground Hardware-In-the-Loop (HIL) experimentation. Likewise, much of the HIL experimentation required for on-orbit servicing of traditional spacecraft systems can also be accomplished in ground-based laboratories (Creamer, 2007). This type of HIL experimentation complements analytical methods and numerical simulations by providing a low-risk, relatively low-cost and potentially highreturn method for validating the technology, navigation techniques and control approaches associated with spacecraft systems. Several approaches exist for the actual HIL testing in a laboratory environment with respect to spacecraft guidance, navigation and control. One 
such method involves reproduction of the kinematics and vehicle dynamics for 3-DoF (two horizontal translational degrees and one rotational degree about the vertical axis) through the use of robotic spacecraft simulators that float via planar air bearings on a flat horizontal floor. This particular method is currently being employed by several research institutions and is the validation method of choice for our research into GNC algorithms for proximity operations at the Naval Postgraduate School (Machida et al., 1992; Ullman, 1993; Corrazzini \& How, 1998; Marchesi et al., 2000; Ledebuhr et al., 2001; Nolet et al., 2005; LeMaster et al., 2006; Romano et al., 2007). With respect to spacecraft involved in proximity operations, the in-plane and cross-track dynamics are decoupled, as modeled by the Hill-ClohessyWiltshire (HCW) equations, thus the reduction to 3-Degree of Freedom (DoF) does not appear to be a critical limiter. One consideration involves the reduction of the vehicle dynamics to one of a double integrator. However, the orbital dynamics can be considered to be a disturbance that needs to be compensated for by the spacecraft navigation and control system during the proximity navigation and assembly phase of multiple systems. Thus the flat floor testbed can be used to capture many of the critical aspects of an actual autonomous proximity maneuver that can then be used for validation of numerical simulations. Portions of the here-in described testbed, combined with the first generation robotic spacecraft simulator of the Spacecraft Robotics Laboratory (SRL) at Naval Postgraduate School (NPS), have been employed to propose and experimentally validate control algorithms. The interested reader is referred to (Romano et al., 2007) for a full description of this robotic spacecraft simulator and the associated HIL experiments involving its demonstration of successful autonomous spacecraft approach and docking maneuvers to a collaborative target with a prototype docking interface of the Orbital Express program.

Given the requirement for spacecraft aggregates to rendezvous and dock during the final phases of assembly and a desire to maximize the useable surface area of the spacecraft for power generation, sensor packages, docking mechanisms and payloads while minimizing thruster impingement, control of such systems using the standard control actuator configuration of fixed thrusters on each face coupled with momentum exchange devices can be challenging if not impossible. For such systems, a new and unique configuration is proposed which may capitalize, for instance, on the recently developed carpal robotic joint invented by Dr. Steven Canfield with its hemispherical vector space (Canfield, 1998). It is here demonstrated through Lie algebra analytical methods and experimental results that two vectorable in-plane thrusters in an opposing configuration can yield a minimum set of actuators for a controllable system. It will also be shown that by coupling the proposed set of vectorable thrusters with a single degree of freedom Control Moment Gyroscope, an additional degree of redundancy can be gained. Experimental results are included using SRL's second generation reduced order (3 DoF) spacecraft simulator. A general overview of this spacecraft simulator is presented in this chapter (additional details on the simulators can be found in: Hall, 2006; Eikenberry, 2006; Price, W., 2006; Romano \& Hall, 2006; Hall \& Romano, 2007a; Hall \& Romano, 2007b).

While presenting an overview of a robotic testbed for HIL experimentation of guidance and control algorithms for on-orbit proximity maneuvers, this chapter specifically focuses on exploring the feasibility, design and evaluation in a 3-DoF environment of a vectorable thruster configuration combined with optional miniature single gimbaled control moment gyro (MSGCMG) for an agile small spacecraft. Specifically, the main aims are to present and practically confirm the theoretical basis of small-time local controllability for this unique 
actuator configuration through both analytical and numerical simulations performed in previous works (Romano \& Hall, 2006; Hall \& Romano, 2007a; Hall \& Romano, 2007b) and to validate the viability of using this minimal control actuator configuration on a small spacecraft in a practical way. Furthermore, the experimental work is used to confirm the controllability of this configuration along a fully constrained trajectory through the employment of a smooth feedback controller based on state feedback linearization and linear quadratic regulator techniques and proper state estimation methods. The chapter is structured as follows: First the design of the experimental testbed including the floating surface and the second generation 3-DoF spacecraft simulator is introduced. Then the dynamics model for the spacecraft simulator with vectorable thrusters and momentum exchange device are formulated. The controllability concerns associated with this uniquely configured system are then addressed with a presentation of the minimum number of control inputs to ensure small time local controllability. Next, a formal development is presented for the state feedback linearized controller, state estimation methods, Schmitt trigger and Pulse Width Modulation scheme. Finally, experimental results are presented.

\section{The NPS Robotic Spacecraft Simulator Testbed}

Three generations of robotic spacecraft simulators have been developed at the NPS Spacecraft Robotics Laboratory, in order to provide for relatively low-cost HIL experimentation of GNC algorithms for spacecraft proximity maneuvers (see Fig.1). In particular, the second generation robotic spacecraft simulator testbed is used for the here-in presented research. The whole spacecraft simulator testbed consists of three components. The two components specifically dedicated to HIL experimentation in 3-DoF are a floating surface with an indoor pseudo-GPS (iGPS) measurement system and one 3-DoF autonomous spacecraft simulator. The third component of the spacecraft simulator testbed is a 6-DoF simulator stand-alone computer based spacecraft simulator and is separated from the HIL components. Additionally, an off-board desktop computer is used to support the 3DoF spacecraft simulator by providing the capability to upload software, initiate experimental testing, receive logged data during testing and process the iGPS position coordinates. Fig. 2 depicts the robotic spacecraft simulator in the Proximity Operations Simulator Facility (POSF) at NPS with key components identified. The main testbed systems are briefly described in the next sections with further details given in (Hall, 2006; Price, 2006; Eikenberry, 2006; Romano \& Hall, 2006; Hall \& Romano, 2007a; Hall \& Romano 2007b). 


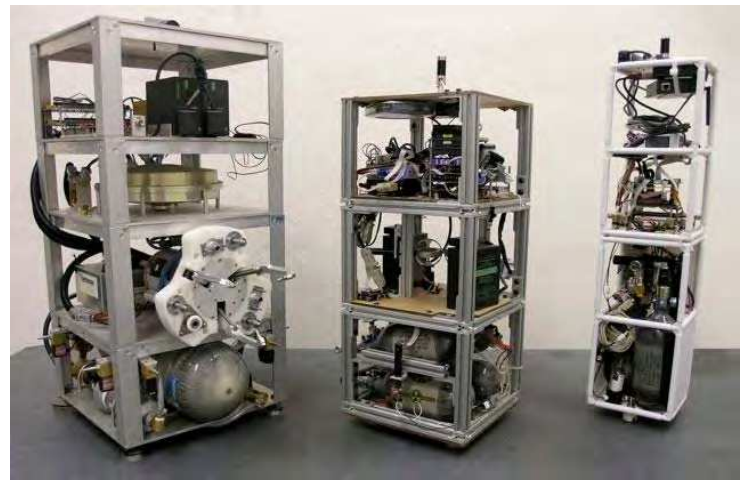

Fig. 1. Three generations of spacecraft simulator at the NPS Spacecraft Robotics Laboratory (first, second and third generations from left to right)

\subsection{Floating Surface}

A $4.9 \mathrm{~m}$ by $4.3 \mathrm{~m}$ epoxy floor surface provides the base for the floatation of the spacecraft simulator. The use of planar air bearings on the simulator reduces the friction to a negligible level and with an average residual slope angle of approximately $2.6 \times 10^{-3} \mathrm{deg}$ for the floating surface, the average residual acceleration due to gravity is approximately $1.8 \times 10^{-3} \mathrm{~ms}^{-2}$. This value of acceleration is 2 orders of magnitude lower than the nominal amplitude of the measured acceleration differences found during reduced gravity phases of parabolic flights (Romano et al, 2007).

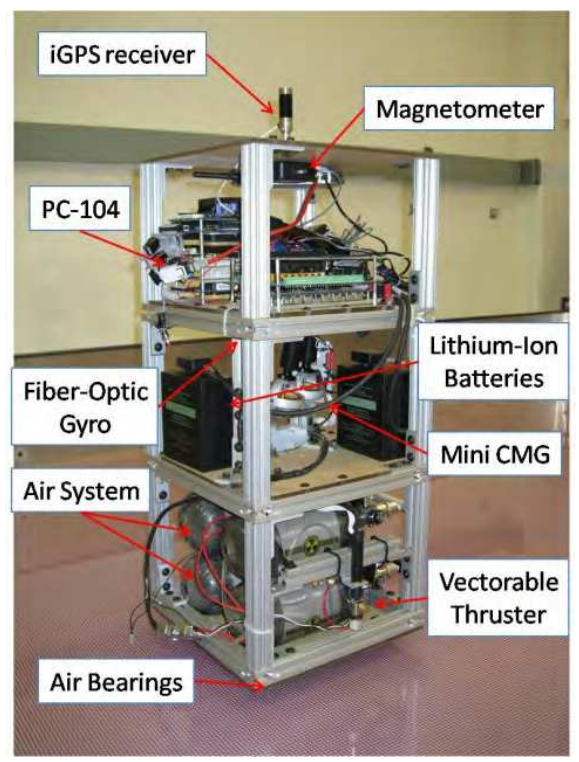

Fig. 2. SRL's 2nd Generation 3-DoF Spacecraft Simulator 


\subsection{3-DoF Robotic Spacecraft Simulator}

SRL's second generation robotic spacecraft simulator is modularly constructed with three easily assembled sections dedicated to each primary subsystem. Prefabricated 6105-T5 Aluminum fractional t-slotted extrusions form the cage of the vehicle while one square foot, .25 inch thick static dissipative rigid plastic sheets provide the upper and lower decks of each module. The use of these materials for the basic structural requirements provides a high strength to weight ratio and enable rapid assembly and reconfiguration. Table 1 reports the key parameters of the 3-DoF spacecraft simulator.

\subsubsection{Propulsion and Flotation Subsystems}

The lowest module houses the flotation and propulsion subsystems. The flotation subsystem is composed of four planar air bearings, an air filter assembly, dual 4500 PSI (31.03 MPa) carbon-fiber spun air cylinders and a dual manifold pressure reducer to provide 75 PSI (.51 $\mathrm{MPa})$. This pressure with a volume flow rate for each air bearing of $3.33 \mathrm{slfm}\left(3.33 \times 10^{-3}\right.$ $\mathrm{m}^{3} / \mathrm{min}$ ) is sufficient to keep the simulator in a friction-free state for nearly 40 minutes of continuous experimentation time. The propulsion subsystem is composed of dual vectorable supersonic on-off cold-gas thrusters and a separate dual carbon-fiber spun air cylinder and pressure reducer package regulated at $60 \mathrm{PSI}(.41 \mathrm{MPa})$ and has the capability of providing the system $31.1 \mathrm{~m} / \mathrm{s} \Delta V$.

\subsubsection{Electronic and Power Distribution Subsystems}

The power distribution subsystem is composed of dual lithium-ion batteries wired in parallel to provide 28 volts for up to 12 Amp-Hours and is housed in the second deck of the simulator. A four port DC-DC converter distributes the requisite power for the system at 5 , 12 or 24 volts DC. An attached cold plate provides heat transfer from the array to the power system mounting deck in the upper module. The current power requirements include a single PC-104 CPU stack, a wireless router, three motor controllers, three separate normallyclosed solenoid valves for thruster and air bearing actuation, a fiber optic gyro, a magnetometer and a wireless server for transmission of the vehicle's position via the pseudo-GPS system. 


\begin{tabular}{lll}
\hline \hline Subsystem & Characteristic & Parameter \\
\hline Structure & Length and width & $.30 \mathrm{~m}$ \\
& Height & $.69 \mathrm{~m}$ \\
& Mass (Overall) & $26 \mathrm{~kg}$ \\
& $J_{z}$ (Overall) & $.40 \mathrm{~kg}-\mathrm{m}^{2}$ \\
\hline Propulsion & Propellant & Compressed Air \\
& Equiv. storage capacity & $.05 \mathrm{~m}^{3} @ 31.03 \mathrm{Mpa}$ \\
& Operating pressure & $.41 \mathrm{Mpa}$ \\
& Thrust (x2) & $.159 \mathrm{~N}$ \\
& ISP & $34.3 \mathrm{~s}$ \\
& Total $\Delta V$ & $31.1 \mathrm{~m} / \mathrm{s}$ \\
\hline Flotation & Propellant & Air \\
& Equiv. storage capacity & $0.05 \mathrm{~m}{ }^{3} @ 31.03 \mathrm{Mpa}$ \\
& Operating pressure & $.51 \mathrm{Mpa}$ \\
& Linear air bearing (x4) & $32 \mathrm{~mm}$ diameter \\
& Continuous operation & $\sim 40 \mathrm{~min}$ \\
\hline CMG Attitude Control & Max torque & $.668 \mathrm{Nm}$ \\
& Momentum storage & $.098 \mathrm{Nms}$ \\
\hline Electrical \& Electronic & Battery type & Lithium-Ion \\
& Storage capacity & $12 \mathrm{Ah} @ 28 \mathrm{~V}$ \\
& Continuous Operation & $\sim 6 \mathrm{~h}$ \\
& Computer & 1 PC104 Pentium III \\
\hline Sensors & Fiber optic gyro & KVH Model DSP-3000 \\
& Position sensor & Metris iGPS \\
& Magnetometer & MicroStrain 3DM-GX1 \\
\hline \hline
\end{tabular}

Table 1. Key Parameters of the 2nd generation 3-DoF Robotic Spacecraft Simulator

\subsubsection{Translation and Attitude Control System Actuators}

The 3-DoF robotic spacecraft simulator includes actuators to provide both translational control and attitude control. A full development of the controllability for this unique configuration of dual rotating thrusters and one-axis Miniature-Single Gimbaled Control Moment Gyro (MSGCMG) will be demonstrated in subsequent sections of this paper. The translational control is provided by two cold-gas on-off supersonic nozzle thrusters in a dual vectorable configuration. Each thruster is limited in a region $\pm \pi / 2$ with respect to the face normal and, through experimental testing at the supplied pressure, has been demonstrated to have an ISP of $34.3 \mathrm{~s}$ and able to provide $.159 \mathrm{~N}$ of thrust with less than 10 msec actuation time (Lugini, 2008). The MSGCMG is capable of providing .668 Nm of torque with a maximum angular momentum of $.098 \mathrm{Nms}$.

\subsection{6-DoF Computer-Based Numerical Spacecraft Simulator}

A separate component of SRL's spacecraft simulator testbed at NPS is a 6-DoF computerbased spacecraft simulator. This simulator enables full 6-DoF numerical simulations to be conducted with realistic orbital perturbations including aerodynamic, solar pressure and 
third-body effects, and earth oblateness up to J4. Similar to the 3-DoF robotic simulator, the numerical simulator is also modularly designed within a MATLAB®/Simulink $®$ architecture to allow near seamless integration and testing of developed guidance and control algorithms. Additionally, by using the MATLAB®/Simulink ${ }^{\circledR}$ architecture with the added Real Time Workshop ${ }^{\mathrm{TM}}$ toolbox, the developed control algorithms can be readily transitioned into C-code for direct deployment onto the 3-DoF robotic simulator's onboard processor. A full discussion of the process by which this is accomplished and simplified for rapid real-time experimentation on the 3-DoF testbed for either the proprietary MATLAB ${ }^{\circ}$ based XPCTarget ${ }^{\mathrm{TM}}$ operating system is given in (Hall, 2006; Price, 2006) or for an opensource Linux based operating system with the Real Time Application Interface (RTAI) is given in (Bevilacqua et al., 2009).

\section{Dynamics of a 3-DoF Spacecraft Simulator with Vectorable Thrusters and Momentum Exchange Device}

Two sets of coordinate frame are established for reference: the inertial coordinate system (ICS) designated by XYZ and body-fixed coordinate system (BCS) designated by $x y z$. These reference frames are depicted in Fig. 3 along with the necessary external forces and parameters required to properly define the simulators motion. The origin of the body-fixed coordinate system is taken to be the center of mass $C$ of the spacecraft simulator and this is assumed to be collocated with the simulator's geometric center. The body $z$-axis is aligned with the inertial $Z$-axis while the body $x$-axis is in line with the thrusters points of action. In the ICS, the position and velocity vectors of $C$ are given by $\mathbf{X}$ and $\mathbf{V}$ so that $\mathbf{X}(X, Y)$ marks the position of the simulator with respect to the origin of the ICS as measured by the inertial measurement sensors and provides the vehicle's two degrees of translational freedom. The vehicle's rotational freedom is described by an angle of rotation $\psi$ between the $x$-axis and the $X$-axis about the $z$-axis. The angular velocity is thus limited to one degree of freedom and is denoted by $\omega_{z}$. The spacecraft simulator is assumed to be rigid and therefore a constant moment of inertia $\left(J_{z}\right)$ exists about the $z$-axis. Furthermore, any changes to the mass of the simulator $(m)$ due to thruster firing are neglected.

The forces imparted at a distance $L$ from the center of mass by the vectorable on-off thrusters are denoted by $F_{1}$ and $F_{2}$ respectively. The direction of the thrust vector $\mathbf{F}_{1}$ is determined by $\alpha_{1}$ which is the angle measured from the outward normal of face one in a clockwise direction (right-hand rotation) to where thruster one's nozzle is pointing. Likewise, the direction of the thrust vector $\mathbf{F}_{2}$ is determined by $\alpha_{2}$ which is the angle measured from the outward normal of face two in a clockwise direction (right-hand rotation) to where thruster two's nozzle is pointing. The torque imparted on the vehicle by a momentum exchange device such as a control moment gyro is denoted by $T_{M E D}$ and can be constrained to exist only about the yaw axis as demonstrated in (Hall, 2006; Romano \& Hall, 2006). 


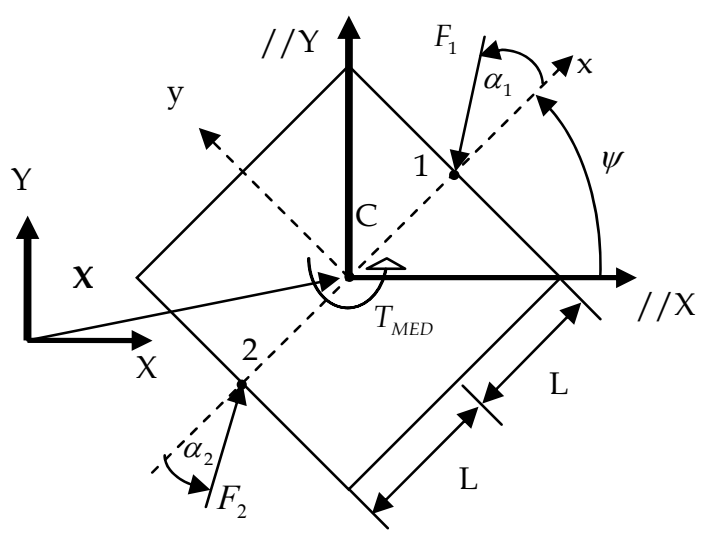

Fig. 3. SRL's 2nd Generation Spacecraft Simulator Schematic

The translation and attitude motion of the simulator are governed by the equations

$$
\begin{aligned}
& \dot{\mathbf{X}}=\mathbf{V} \\
& \dot{\mathbf{V}}=m^{-1{ }^{I I}} \mathbf{R}_{B}(\psi){ }^{B} \mathbf{F} \\
& \dot{\psi}=\omega_{z} \\
& \dot{\omega}_{z}=J_{z}^{-1}{ }^{B} T
\end{aligned}
$$

where ${ }^{B} \mathbf{F} \in \mathbb{R}^{2}$ are the thruster inputs limited to the region $\pm \pi / 2$ with respect to each face normal and ${ }^{B} T \in \mathbb{R}$ is the attitude input. ${ }^{I} \mathbf{R}_{B}(\psi),{ }^{B} \mathbf{F}$ and ${ }^{B} T$ are given by

$$
\begin{gathered}
{ }^{I} \mathbf{R}_{B}(\psi)=\left[\begin{array}{cc}
\mathrm{c} \psi & -\mathrm{s} \psi \\
\mathrm{s} \psi & \mathrm{c} \psi
\end{array}\right] \\
{ }^{B} \mathbf{F}^{T}={ }^{B} \mathbf{F}_{1}^{T}+{ }^{B} \mathbf{F}_{2}^{T}=\left[-F_{1} \mathrm{c} \alpha_{1}+F_{2} \mathrm{c} \alpha_{2},-F_{1} \mathrm{~s} \alpha_{1}+F_{2} \mathrm{~s} \alpha_{2}\right]^{T} \\
{ }^{B} T=\left[T_{M E D}+L\left(-F_{1} \mathrm{~s} \alpha_{1}-F_{2} \mathrm{~s} \alpha_{2}\right)\right]
\end{gathered}
$$

where $\mathrm{s} \bullet=\sin (\bullet), \mathrm{c} \bullet=\cos (\bullet)$.

The internal dynamics of the vectorable thrusters are assumed to be linear according to the following equations

$$
\dot{\alpha}_{1}=\beta_{1}, \dot{\beta}_{1}=J_{1}^{-1} T_{1}, \dot{\alpha}_{2}=\beta_{2}, \dot{\beta}_{2}=J_{2}^{-1} T_{2}
$$

where $J_{1}$ and $J_{2}$ represent the moments of inertia about each thruster rotational axis respectively and $T_{1} \in \mathbb{R}, T_{2} \in \mathbb{R}$ represent the corresponding thruster rotation control input. The system's state equation given by Eq. (1) can be rewritten in control-affine system form as (LaValle, 2006) 


$$
\dot{\mathbf{x}}=\mathbf{f}(\mathbf{x})+\sum_{i=1}^{N_{u}} u_{i} \mathbf{g}_{i}(\mathbf{x})=\mathbf{f}(\mathbf{x})+G(\mathbf{x}) \mathbf{u}, x \in \mathbb{R}^{N_{x}}
$$

where $N_{u}$ is the number of controls. With $\mathbb{R}^{N_{x}}$ representing a smooth $N_{x}$-dimensional manifold defined be the size of the state-vector and the control vector to be in $\mathbb{R}^{N_{u}}$. Defining the state vector $\mathbf{x} \in \mathbb{R}^{10}$ as $\mathbf{x}^{T}=\left[x_{1}, x_{2}, \ldots, x_{10}\right]=\left[X, Y, \psi, \alpha_{1}, \alpha_{2}, V_{x}, V_{y}, \omega_{z}, \beta_{1}, \beta_{2}\right]$ and the control vector $\mathbf{u} \in \mathrm{U}^{5}$ as $\mathbf{u}^{T}=\left[u_{1}, u_{2}, \ldots, u_{5}\right]=\left[F_{1}, F_{2}, T_{M E D}, T_{1}, T_{2}\right]$, the system's state equation, becomes

$$
\dot{\mathbf{x}}=\mathbf{f}(\mathbf{x})+G(\mathbf{x}) \mathbf{u}=\left[x_{6}, x_{7}, x_{8}, x_{9}, x_{10}, \mathbf{0}_{1 \times 5}\right]^{T}+\left[\begin{array}{c}
\mathbf{0}_{5 x 5} \\
G_{1}(\mathbf{x})
\end{array}\right] \mathbf{u}
$$

where the matrix $G_{1}(\mathbf{x})$ is obtained from Eq. (1) as

$$
G_{1}(\mathbf{x})=\left[\begin{array}{ccccc}
-m^{-1}\left[\mathrm{c} x_{3} \mathrm{c} x_{4}-\mathrm{s} x_{3} \mathrm{~s} x_{4}\right] & m^{-1}\left[\mathrm{c} x_{3} \mathrm{c} x_{5}-\mathrm{s} x_{3} \mathrm{~s} x_{5}\right] & 0 & 0 & 0 \\
-m^{-1}\left[\mathrm{c} x_{3} \mathrm{~s} x_{4}+\mathrm{s} x_{3} \mathrm{c} x_{4}\right] & m^{-1}\left[\mathrm{c} x_{3} \mathrm{~s} x_{5}+\mathrm{s} x_{3} \mathrm{c} x_{5}\right] & 0 & 0 & 0 \\
-J_{z}^{-1} \mathrm{~s} x_{4} L & -J_{z}^{-1} \mathrm{~s} x_{5} L & J_{z}^{-1} & 0 & 0 \\
0 & 0 & 0 & J_{2}^{-1} & 0 \\
0 & 0 & 0 & 0 & J_{2}^{-1}
\end{array}\right]
$$

With the system in the form of Eq. (6) given the vector fields in Eqs. (7) and (8), and given that $\mathbf{f}(\mathbf{x})$ (the drift term) and $G(\mathbf{x})$ (the control matrix of control vector fields) are smooth functions, it is important to note that it is not necessarily possible to obtain zero velocity due to the influence of the drift term. This fact places the system in the unique subset of controlaffine systems with drift and, as seen later, will call for an additional requirement for determining the controllability of the system. Furthermore, when studying controllability of systems, the literature to date restricts the consideration to cases where the control is proper. Having a proper control implies that the affine hull of the control space is equal to $\mathbb{R}^{N_{u}}$ or that the smallest subspace of $U$ is equal to the number of control vectors and that it is closed (Sussman, 1987; Sussman, 1990; Bullo \& Lewis, 2005; LaValle, 2006). With a system such as a spacecraft in general or the simplified model of the 3-DoF simulator in particular, the use of on-off cold-gas thrusters restrict the control space to only positive space with respect to both thrust vectors leading to an unclosed set and thus improper control space. In order to overcome this issue, a method which leverages the symmetry of the system is used by which the controllability of the system is studied by considering only one virtual rotating thruster that is positioned a distance $L$ from the center of mass with the vectored thrust resolved into a $\mathrm{y}$ and $\mathrm{x}$-component. In considering this system perspective, the thruster combination now spans $\mathbb{R}^{2}$ and therefore is proper and is analogous to the planar body with variable-direction force vector considered in (Lewis \& Murray, 1997; Bullo \& Lewis, 2005). Furthermore, under the assumption that the control bandwidth of the thrusters's rotation is much larger than the control bandwidth of the system dynamics, the internal dynamics of the vectorable thrusters can be decoupled from the state and control vectors for the system yielding a thrust vector dependent on simply a commanded angle. Thus the system's state vector, assuming that both thrusters and a momentum exchange device are available, 
becomes $\quad \mathbf{x}^{T}=\left[x_{1}, x_{2}, \ldots, x_{6}\right]=\left[X, Y, \psi, V_{X}, V_{Y}, \omega_{z}\right] \in \mathbb{R}^{6}$ and the control vector is $\mathbf{u}^{T}=\left[u_{1}, u_{2}, u_{3}\right]=\left[{ }^{B} F_{x},{ }^{B} F_{y}, T_{z}\right]=U^{3}$ so that the system's state equation becomes

$$
\dot{\mathbf{x}}=\mathbf{f}(\mathbf{x})+G(\mathbf{x}) \mathbf{u}=\left[x_{4}, x_{5}, x_{6}, 0,0,0\right]^{T}+\left[\begin{array}{c}
\mathbf{0}_{3 x 3} \\
G_{1}(\mathbf{x})
\end{array}\right] \mathbf{u}
$$

where the matrix $G_{1}(\mathbf{x})$ can be obtained by considering the relation of the desired control vector to the body centered reference system, in the two cases of positive force needed in the $x$ direction $\left({ }^{B} U_{x}>0\right)$ and negative force needed in the $x$ direction $\left({ }^{B} U_{x}<0\right)$. In this manner, the variables in Eq. (8) and Eq. (9) can be defined as

$$
\begin{aligned}
& { }^{B} U_{x}<0 \rightarrow\left\{\begin{array}{l}
\mathbf{u}^{T}=\left[{ }^{B} F_{x},{ }^{B} F_{y},{ }^{B} T_{z}\right]=\left[-F_{1} \mathrm{c} x_{4},-F_{1} \mathrm{~s} x_{4}, T_{M E D}\right] \\
d=-L, F_{2}=0
\end{array}\right. \\
& { }^{B} U_{x}>0 \rightarrow\left\{\begin{array}{l}
\mathbf{u}^{T}=\left[{ }^{B} F_{x},{ }^{B} F_{y},{ }^{B} T_{z}\right]=\left[F_{2} \mathrm{c} x_{5}, F_{2} \mathrm{~s} x_{5}, T_{M E D}\right] \\
d=L, F_{1}=0
\end{array}\right.
\end{aligned}
$$

yielding the matrix in $G_{1}(\mathbf{x})$ through substitution into Eq. (8) as

$$
G_{1}(\mathbf{x})=\left[\begin{array}{ccc}
m^{-1} \mathrm{c} x_{3} & -m^{-1} \mathrm{~s} x_{3} & 0 \\
m^{-1} \mathrm{~s} x_{3} & m^{-1} \mathrm{c} x_{3} & 0 \\
0 & -d J_{z}^{-1} & J_{z}^{-1}
\end{array}\right]
$$

When the desired control input to the system along the body $x$-axis is zero, both thrusters can be used to provide a control force along the $y$-axis, while a momentum exchange device provides any required torque. In this case, the control vector in (9) becomes $\mathbf{u}^{T}=\left[u_{1}, u_{2}\right]=\left[{ }^{B} F_{y},{ }^{B} T_{z}\right]=U^{2}$ such that the variables in Eq. (8) and (9) can be defined as

$$
{ }^{B} U_{x}=0 \rightarrow\left\{\begin{array}{l}
\mathbf{u}^{T}=\left[{ }^{B} F_{y}, T_{z}\right]=\left[F s \alpha, T_{M E D}\right] \\
F=F_{1}=F_{2}, \alpha=x_{4}=-x_{5}=-\frac{\pi}{2} \operatorname{sign}\left({ }^{B} U_{y}\right)
\end{array}\right.
$$

which yields the matrix $G_{1}(\mathbf{x})$ through substitution into Eq. (8) as

$$
G_{1}(\mathbf{x})=\left[\begin{array}{cc}
-2 m^{-1} \mathrm{~s}_{3} & 0 \\
2 m^{-1} \mathrm{c} x_{3} & 0 \\
0 & J_{z}^{-1}
\end{array}\right]
$$


As will be demonstrated in later, the momentum exchange device is not necessary to ensure small time controllability for this system. In considering this situation, which also occurs when a control moment gyroscope is present but is near the singular conditions and therefore requires desaturation, the thruster not being used for translation control can be slewed to $\pm \pi / 2$ depending on the required torque compensation and fired to affect the desired angular rate change. The desired control input to the system with respect to the body $\mathrm{x}$-axis $\left({ }^{B} U_{x}\right)$ can again be used to define the desired variables such that

$$
\begin{aligned}
& { }^{B} U_{x} \leq 0 \rightarrow\left\{\begin{array}{l}
\mathbf{u}^{T}=\left[{ }^{B} F_{x},{ }^{B} F_{y},{ }^{B} T_{z}\right]=\left[-F_{1} \mathrm{c} x_{4},-F_{1} \mathrm{~s} x_{4}, F_{2} d \mathrm{~s} x_{5}\right] \\
d=-L, x_{5}= \pm \frac{\pi}{2}
\end{array}\right. \\
& { }^{B} U_{x}>0 \rightarrow\left\{\begin{array}{l}
\mathbf{u}^{T}=\left[{ }^{B} F_{x},{ }^{B} F_{y}, T_{z}\right]=\left[F_{2} \mathrm{c} x_{5}, F_{2} \mathrm{~s} x_{5},-F_{1} d \mathrm{~s} x_{4}\right] \\
d=L, x_{4}= \pm \frac{\pi}{2}
\end{array}\right.
\end{aligned}
$$

which yields the matrix $G_{1}(\mathbf{x})$ through substitution into Eq. (8)as

$$
G_{1}(\mathbf{x})=\left[\begin{array}{ccc}
m^{-1} \mathrm{c} x_{3} & -m^{-1} \mathrm{~s} x_{3} & -(m d)^{-1} \mathrm{~s} x_{3} \\
m^{-1} \mathrm{~s} x_{3} & m^{-1} \mathrm{c} x_{3} & (m d)^{-1} \mathrm{c} x_{3} \\
0 & -d J_{z}^{-1} & J_{z}^{-1}
\end{array}\right]
$$

In case of zero force requested along $\mathrm{x}$ with only thrusters acting, the system cannot in general provide the requested torque value.

A key design consideration with this type of control actuator configuration is that with only the use of an on/off rotating thruster to provide the necessary torque compensation, fine pointing can be difficult and more fuel is required to affect a desired maneuver involving both translation and rotation.

\section{Small-Time Local Controllability}

Before studying the controllability for a nonlinear control-affine system of the form in Eq. (6), it is important to review several definitions. First, the set of states reachable in time at most $T$ is given by $R_{\Sigma}\left(\mathbf{x}_{0}, \leq T\right)$ by solutions of the nonlinear control-affine system.

Definition 1 (Accessibility)

A system is accessible from $\mathbf{x}_{0}$ (the initial state) if there exists $T>0$ such that the interior of $R_{\Sigma}\left(\mathbf{x}_{0}, \leq t\right)$ is not an empty set for $\left.\left.t \in\right] 0, T\right]$ (Bullo \& Lewis, 2005).

Definition 2 (Proper Small Time Local Controllability)

A system is small time locally controllable (STLC) from $\mathbf{x}_{0}$ if there exists $T>0$ such that $\mathbf{x}_{0}$ lies in the interior of $R_{\Sigma}\left(\mathbf{x}_{0}, \leq t\right)$ for each $\left.\left.t \in\right] 0, T\right]$ for every proper control set $U$ (Bullo \& 
Lewis, 2005). Assuming that at $\mathbf{x}(0)=\mathbf{0}$ this can also be seen under time reversal as the equilibrium for the system $x_{0}$ can be reached from a neighborhood in small time (Sussman, 1987; Sussman, 1990).

Definition 3 (Proper Control Set) A control set $\mathbf{u}^{T}=\left[u_{1}, \ldots, u_{k}\right]$ is termed to be proper if the set satisfies a constraint $\mathbf{u} \in K$ where $K$ affinely spans $U^{k}$. (Sussman, 1990; Bullo \& Lewis, 2005; LaValle, 2006).

Definition 4 (Lie derivative) The Lie derivative of a smooth scalar function $\mathrm{g}(\mathbf{x}) \in \mathbb{R}$ with respect to a smooth vector field $\mathbf{f}(\mathbf{x}) \in \mathbb{R}^{N_{x}}$ is a scalar function defined as (Slotine, 1991, pg. 229)

$$
L_{\mathbf{f}} g=\nabla g \mathbf{f}=\left[\frac{\partial g}{\partial x_{1}} \ldots \frac{\partial g}{\partial x_{N_{x}}}\right]\left[\begin{array}{c}
f_{1}(\mathbf{x}) \\
\vdots \\
f_{N_{x}}(\mathbf{x})
\end{array}\right] .
$$

Definition 5 (Lie Bracket): The Lie bracket of two vector fields $\mathbf{f}(\mathbf{x}) \in \mathbb{R}^{N_{x}}$ and $\mathbf{g}(\mathbf{x}) \in \mathbb{R}^{N_{x}}$ is a third vector field $[\mathbf{f}, \mathbf{g}] \in \mathbb{R}^{N_{x}}$ defined by $[\mathbf{f}, \mathbf{g}]=\nabla \mathbf{g} \mathbf{f}-\nabla \mathbf{f} \mathbf{g}$, where the $i$-th component can be expressed as (Slotine, 1991)

$$
[\mathbf{f}, \mathbf{g}]_{i}=\sum_{j=1}^{N_{x}}\left(f_{j} \frac{\partial g_{i}}{\partial x_{j}}-g_{j} \frac{\partial f_{i}}{\partial x_{j}}\right)
$$

Using Lie bracketing methods which produce motions in directions that do not seem to be allowed by the system distribution, sufficient conditions can be met to determine a system's STLC even in the presence of a drift vector as in the equations of motion developed above. These sufficient conditions involve the Lie Algebra Rank Condition (LARC).

Definition 6 (Associated Distribution $\Delta(\mathbf{x})$ ) Given a system as in Eq. (6), the associated distribution $\Delta(\mathbf{x})$ is defined as the vector space (subspace of $\mathbb{R}^{N_{x}}$ ) spanned by the system vector fields $\mathbf{f}, \mathbf{g}_{1}, \ldots \mathbf{g}_{N_{u}}$.

Definition 7 The Lie algebra of the associated distribution $\mathcal{L}(\Delta)$ is defined to be the distribution of all independent vector fields that can be obtained by applying subsequent Lie bracket operations to the system vector fields. Of note, no more than $N_{x}$ vector fields can be produced (LaValle, 2006). With $\operatorname{dim}(\mathcal{L}(\Delta)) \leq N_{x}$, the computation of the elements of $\mathcal{L}(\Delta)$ ends either when $N_{x}$ independent vector fields are obtained or when all subsequent Lie brackets are vector fields of zeros.

Definition 8 (Lie Algebra Rank Condition (LARC)) The Lie Algebra Rank Condition is satisfied at a state $\mathbf{x}$ if the rank of the matrix obtained by concatenating the vector fields of the Lie algebra distribution at $\mathbf{x}$ is equal to $N_{x}$ (the number of state).

For a driftless control-affine system, following the Chow-Rashevskii Theorem, the system is STLC if the LARC is satisfied (Lewis \& Murray, 1997; Bullo \& Lewis, 2005; LaValle, 2006). However, given a system with drift, in order to determine the STLC, the satisfaction of the 
LARC it is not sufficient: in addition to the LARC, it is necessary to examine the combinations of the vectors used to compose the Lie brackets of the Lie algebra. From Sussman's General Theorem on Controllability, if the LARC is satisfied and if there are no ill formed brackets in $\mathcal{L}(\Delta)$, then the system is STLC from its equilibrium point (Sussman, 1987). The Sussman's theorem, formally stated is reported here below.

Theorem 1 (Sussman's General Theorem on Controllability) Consider a system given by Eq. (6) and an equilibrium point $\mathbf{p} \in \mathbb{R}^{N_{x}}$ such that $\mathbf{f}(\mathbf{p})=\mathbf{0}$. Assume $\mathcal{L}(\Delta)$ satisfies the LARC at $\mathbf{p}$. Furthermore, assume that whenever a potential Lie bracket consists of the drift vector $\mathbf{f}(\mathbf{x})$ appearing an odd number of times while $\mathbf{g}_{1}(\mathbf{x}), \ldots, \mathbf{g}_{N_{u}}(\mathbf{x})$ all appear an even number of times to include zero times (indicating an ill formed Lie bracket), there are sufficient successive Lie brackets to overcome this ill formed Lie bracket to maintain LARC. Then the system is STLC from p . (Sussman, 1987; Sussman, 1990).

As it is common in literature, an ill formed bracket is dubbed a "bad" bracket (Sussman, 1987; Sussman, 1990; Lewis \& Murray, 1997, Bullo \& Lewis, 2005; LaValle, 2006). Conversely, if a bracket is not "bad", it is termed "good". As an example, for a system with a drift vector and two control vectors, the bracket $\left[\mathbf{f},\left[\mathbf{g}_{1}, \mathbf{g}_{1}\right]\right]$ is bad, as the drift vector occurs only once while the first control vector appears twice and the second control vector appears zero times. Similarly, the bracket $\left[\mathbf{f},\left[\mathbf{f},\left[\mathbf{f}, \mathbf{g}_{1}\right]\right]\right]$ is good as the first control vector appears only once. Therefore, it can be summarized that if the rank of the Lie algebra of a controlaffine system with drift is equal to the number of states and there exist sufficient "good" brackets to overcome the "bad" brackets to reach the required LARC rank, then the system is small time locally controllable.

\subsection{Small-Time Local Controllability Considerations for the 3-DoF Spacecraft Simulator}

The concept of small time local controllability is better suitable than the one of accessibility for the problem of spacecraft rendezvous and docking, as a spacecraft is required to move in any directions in a small interval of time dependent on the control actuator capabilities (e.g. to avoid obstacles). The finite time $T$ can be arbitrary if the control input is taken to be unbounded and proper (Sussman, 1990; Bullo \& Lewis, 2005; LaValle, 2006).

While no theory yet exists for the study of the general controllability for a non-linear system, the STLC from an equilibrium condition can be studied by employing Sussman's theorem. For the case of spacecraft motion, in order to apply Sussman's theorem, we hypothesize that the spacecraft is moving from an initial condition with velocity close to zero (relative to the origin of an orbiting reference frame).

In applying Sussman's General Theorem on Controllability to the reduced system equations of motion presented in Eq. (9) with $G_{1}(\mathbf{x})$ given in Eq. (11), the Lie algebra evaluates to

$$
\mathcal{L}(\Delta)=\operatorname{span}\left\{\mathbf{g}_{1}, \mathbf{g}_{2}, \mathbf{g}_{3}\left[\mathbf{f}, \mathbf{g}_{1}\right],\left[\mathbf{f}, \mathbf{g}_{2}\right],\left[\mathbf{f}, \mathbf{g}_{3}\right]\right\}
$$


so that $\operatorname{dim}(\mathcal{L}(\Delta))=N_{x}=6$. In order to verify that this is the minimum number of actuators required to ensure STLC, the Lie algebra is reinvestigated for each possible combination of controls. The resulting analysis, as summarized in Table 2, demonstrates that the system is STLC from the systems equilibrium point at $\mathbf{x}_{0}=\mathbf{0}$ given either two rotating thrusters in complementary semi-circle planes or fixed thrusters on opposing faces providing a normal force vector to the face in opposing directions and a momentum exchange device about the center of mass. For instance, in considering the case of control inputs ${ }^{B} F_{y},{ }^{B} T_{z}=T_{M E D}$, Eq. (9) becomes

$$
\begin{aligned}
\dot{\mathbf{x}} & =\mathbf{f}(\mathbf{x})+\mathbf{g}_{1}(\mathbf{x}) u_{1}+\mathbf{g}_{2}(\mathbf{x}) u_{2} \\
& =\left[x_{4}, x_{5}, x_{6}, 0,0,0\right]^{T}+\left[0,0,0, m^{-1} s x_{3}, m^{-1} c x_{3}, J_{z}^{-1} L\right]^{T} u_{1}+\left[0,0,0,0,0, J_{z}^{-1}\right]^{T} u_{2}
\end{aligned}
$$

where $\mathbf{u}=\left[u_{1}, u_{2}\right]=\left[{ }^{B} F_{y},{ }^{B} T_{z}\right] \in U^{2}$. The equilibrium point $\mathbf{p}$ such that $\mathbf{f}(\mathbf{p})=\mathbf{0}$ is $\mathbf{p}=\left[x_{1}, x_{2}, x_{3}, 0,0,0\right]^{T}$. The $\mathcal{L}(\Delta)$ is formed by considering the associated distribution $\Delta(\mathbf{x})$ and successive Lie brackets as

$$
\begin{aligned}
& \mathbf{f}, \quad \mathbf{g}_{1}, \quad \mathbf{g}_{2} \\
& {\left[\mathbf{f}, \mathbf{g}_{1}\right], \quad\left[\mathbf{g}_{1}, \mathbf{g}_{2}\right], \quad\left[\mathbf{f}, \mathbf{g}_{2}\right]} \\
& {\left[\mathbf{f},\left[\mathbf{f}, \mathbf{g}_{1}\right]\right], \quad\left[\mathbf{f},\left[\mathbf{g}_{1}, \mathbf{g}_{2}\right]\right], \quad\left[\mathbf{f},\left[\mathbf{f}, \mathbf{g}_{2}\right]\right],} \\
& {\left[\mathbf{g}_{1},\left[\mathbf{f}, \mathbf{g}_{1}\right]\right], \quad\left[\mathbf{g}_{1},\left[\mathbf{g}_{1}, \mathbf{g}_{2}\right]\right], \quad\left[\mathbf{g}_{1},\left[\mathbf{f}, \mathbf{g}_{2}\right]\right],} \\
& {\left[\mathbf{g}_{2},\left[\mathbf{f}, \mathbf{g}_{1}\right]\right], \quad\left[\mathbf{g}_{2},\left[\mathbf{g}_{1}, \mathbf{g}_{2}\right]\right], \quad\left[\mathbf{g}_{2},\left[\mathbf{f}, \mathbf{g}_{2}\right]\right],} \\
& {\left[\mathbf{f},\left[\mathbf{f},\left[\mathbf{f}, \mathbf{g}_{1}\right]\right]\right], \quad\left[\mathbf{f},\left[\mathbf{f},\left[\mathbf{g}_{1}, \mathbf{g}_{2}\right]\right]\right], \quad\left[\mathbf{f},\left[\mathbf{f},\left[\mathbf{f}, \mathbf{g}_{2}\right]\right]\right], \quad\left[\mathbf{f},\left[\mathbf{g}_{1},\left[\mathbf{f}, \mathbf{g}_{1}\right]\right]\right]}
\end{aligned}
$$

The sequence can first be reduced by considering any "bad" brackets in which the drift vector appears an odd number of times and the control vector fields each appear an even number of times to include zero. In this manner the Lie brackets $\left[\mathbf{g}_{1},\left[\mathbf{f}, \mathbf{g}_{1}\right]\right]$ and $\left[\mathbf{g}_{2},\left[\mathbf{f}, \mathbf{g}_{2}\right]\right]$ can be disregarded.

By evaluating each remaining Lie bracket at the equilibrium point $\mathbf{p}$, the linearly independent vector fields can be found as

$$
\begin{aligned}
& \mathbf{g}_{1}=\left[0,0,0,-m^{-1} s x_{3}, m^{-1} c x_{3},-J_{z}^{-1} L\right]^{T} \\
& \mathbf{g}_{2}=\left[0,0,0,0,0, J_{z}^{-1}\right]^{T} \\
& {\left[\mathbf{f}, \mathbf{g}_{1}\right]=\nabla \mathbf{g}_{1} \cdot \mathbf{f}-\nabla \mathbf{f} \cdot \mathbf{g}_{1}=\left[m^{-1} s x_{3},-m^{-1} c x_{3},-J_{z}^{-1} L, 0,0,0\right]^{T}} \\
& {\left[\mathbf{f}, \mathbf{g}_{2}\right]=\nabla \mathbf{g}_{2} \cdot \mathbf{f}-\nabla \mathbf{f} \cdot \mathbf{g}_{2}=\left[0,0,-J_{z}^{-1}, 0,0,0\right]^{T}} \\
& {\left[\mathbf{g}_{1},\left[\mathbf{f}, \mathbf{g}_{2}\right]\right]=\nabla\left[\mathbf{f}, \mathbf{g}_{2}\right] \cdot \mathbf{g}_{1}-\nabla \mathbf{g}_{1} \cdot\left[\mathbf{f}, \mathbf{g}_{2}\right]=\left[0,0,0,-m^{-1} J_{z}^{-1} c x_{3},-m^{-1} J_{z}^{-1} s x_{3}, 0\right]^{T}} \\
& {\left[\mathbf{f},\left[\mathbf{g}_{1},\left[\mathbf{f}, \mathbf{g}_{1}\right]\right]\right]=\nabla\left[\mathbf{g}_{1},\left[\mathbf{f}, \mathbf{g}_{1}\right]\right] \cdot \mathbf{f}-\nabla \mathbf{f} \cdot\left[\mathbf{g}_{1},\left[\mathbf{f}, \mathbf{g}_{1}\right]\right]=\left[2 L m^{-1} J_{z}^{-1} c x_{3}, 2 L m^{-1} J_{z}^{-1} s x_{3}, 0,0,0,0\right]^{T}}
\end{aligned}
$$


Therefore, the Lie algebra comprised of these vector fields is

$$
\mathcal{L}(\Delta)=\operatorname{span}\left\{\mathbf{g}_{1}, \mathbf{g}_{2},\left[\mathbf{f}, \mathbf{g}_{1}\right],\left[\mathbf{f}, \mathbf{g}_{2}\right],\left[\mathbf{g}_{1},\left[\mathbf{f}, \mathbf{g}_{2}\right]\right],\left[\mathbf{f},\left[\mathbf{g}_{1},\left[\mathbf{f}, \mathbf{g}_{1}\right]\right]\right]\right\}
$$

yielding $\operatorname{dim}(\mathcal{L}(\Delta))=N_{x}=6$, and therefore the system is small time locally controllable.

\begin{tabular}{cccc}
\hline \hline Control & Thruster Positions & $\operatorname{dim}(\mathcal{L}(\Delta))$ & Controllability \\
\hline $\mathbf{u}^{T}=\left[{ }^{B} F_{x}, 0,0\right]$ & $\alpha_{1}=\alpha_{2}=0$ & 2 & Inaccessible \\
$\mathbf{u}^{T}=\left[0,{ }^{B} F_{y}, 0\right]$ & $\alpha_{1}=-\alpha_{2}=\mp \pi / 2$ & 2 & Inaccessible \\
$\mathbf{u}^{T}=\left[0,0,{ }^{B} T_{z}\right]$ & NA & 2 & Inaccessible \\
$\mathbf{u}^{T}=\left[0,{ }^{B} F_{y},{ }^{B} T_{z}=F_{j} L s \alpha_{j}\right]$ & $\alpha_{i}=\mp \pi / 2, \alpha_{j}= \pm \pi / 2$ & 5 & Inaccessible \\
$\mathbf{u}^{T}=\left[{ }^{B} F_{x},{ }^{B} F_{y}, 0\right]$ & $\left|\alpha_{1}\right| \leq \pi / 2,\left|\alpha_{2}\right| \leq \pi / 2$ & 6 & STLC \\
$\mathbf{u}^{T}=\left[{ }^{B} F_{x}, 0,{ }^{B} T_{z}\right]$ & $\alpha_{1}=\alpha_{2}=0$ & 6 & STLC \\
$\mathbf{u}^{T}=\left[0,{ }^{B} F_{y},{ }^{B} T_{z}=T_{M E D}\right]$ & $\alpha_{1}=-\alpha_{2}=\mp \pi / 2$ & 6 & STLC \\
\hline \hline
\end{tabular}

Table 2. STLC Analysis for the 3-DoF Spacecraft Simulator

\section{Navigation and Control of the 3-DoF Spacecraft Simulator}

In the current research, the assumption is made that the spacecraft simulator is maneuvering in the proximity of an attitude stabilized target spacecraft and that this spacecraft follows a Keplarian orbit. Furthermore, the proximity navigation maneuvers are considered to be fast with respect to the orbital period. A pseudo-GPS inertial measurement system by Metris, Inc. (iGPS) is used to fix the ICS in the laboratory setting for the development of the state estimation algorithm and control commands. The $X$-axis is taken to be the vector between the two iGPS transmitters with the $Y$ and $Z$ axes forming a right triad through the origin of a reference system located at the closest corner of the epoxy floor to the first iGPS transmitter. Navigation is provided by fusing of the magnetometer data and fiber optic gyro through a discrete Kalman filter to provide attitude estimation and through the use of a linear quadratic estimator to estimate the translation velocities given inertial position measurements. Control is accomplished through the combination of a state feedback linearized based controller, a linear quadratic regulator, Schmitt trigger logic and Pulse Width Modulation using the minimal control actuator configuration of the 3-DoF spacecraft simulator. Fig. 4 reports a block diagram representation of the control system. 


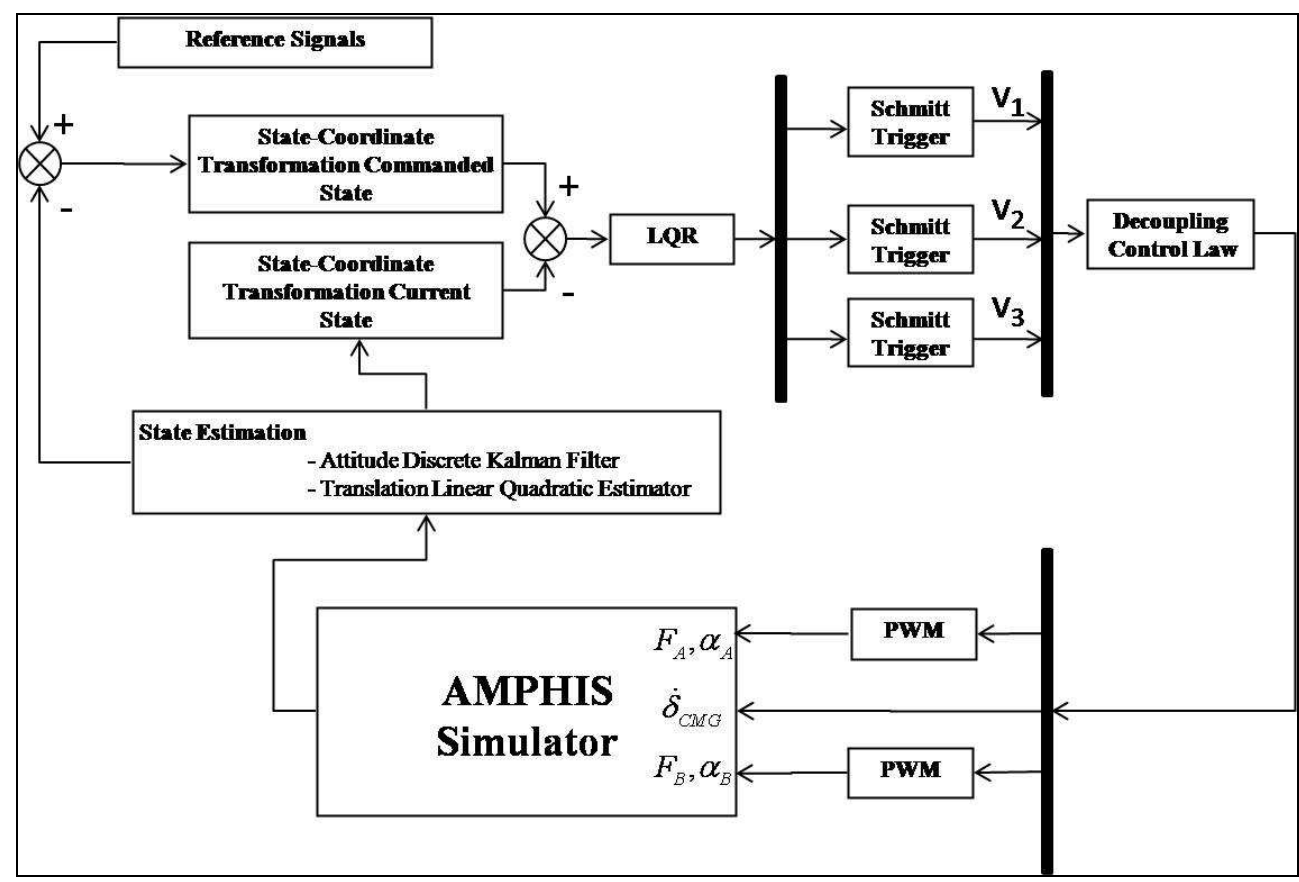

Fig. 4. Block Diagram of the Control System of the 3-DoF Spacecraft simulator

\subsection{Navigation using Inertial Measurements with Kalman Filter and Linear Quadratic Estimator}

In the presence of the high accuracy, low noise, high bandwidth iGPS sensor with position accuracy to within $5.4 \mathrm{~mm}$ with a standard deviation of $3.6 \mathrm{~mm}$ and asynchronous measurement availability with a nominal frequency of $40 \mathrm{~Hz}$, a full-order linear quadratic estimator with respect to the translation states is implemented to demonstrate the capability to estimate the inertial velocities in the absence of accelerometers. Additionally, due to the affect of noise and drift rate in the fiber-optic gyro, a discrete-time linear Kalman filter is employed to fuse the data from the magnetometer and the gyro. Both the gyro and magnetometer are capable of providing new measurements asynchronously at $100 \mathrm{~Hz}$.

\subsubsection{Attitude Discrete-Time Kalman Filter}

With the attitude rate being directly measured, the measurement process can be modeled in state-space equation form as:

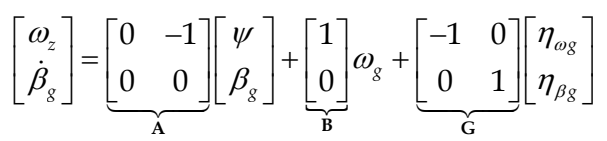

$$
\begin{aligned}
& z=\psi_{m}=\underbrace{\left[\begin{array}{ll}
1 & 0
\end{array}\right]}_{\mathbf{H}}\left[\begin{array}{l}
\psi \\
\beta_{g}
\end{array}\right]+\eta_{\psi m}
\end{aligned}
$$


where $\omega_{g}$ is the measured gyro rate, $\beta_{g}$ is the gyro drift rate, $\eta_{\omega g}$ and $\eta_{\beta g}$ are the associated gyro output measurement noise and the drift rate noise respectively. $\psi_{m}$ is the measured angle from the magnetometer, and $\eta_{\psi m}$ is the associated magnetometer output measurement noise. It is assumed that $\eta_{\omega g}, \eta_{\beta g}$ and $\eta_{\psi m}$ are zero-mean Gaussian whitenoise processes with variances given by $\sigma_{\omega g}^{2}, \sigma_{\beta g}^{2}$ and $\sigma_{\psi m}^{2}$ respectively. Introducing the state variables $\mathbf{x}^{T}=\left[\psi, \beta_{g}\right]$, control variables $u=\omega_{g}$, and error variables $\mathbf{w}^{T}=\left[\eta_{\omega g}, \eta_{\beta g}\right]$ and $v=\eta_{\psi m}$, Eqs. (22) and (23) can be expressed compactly in matrix form as

$$
\begin{gathered}
\dot{\mathbf{x}}(t)=A(t) \mathbf{x}(t)+B(t) \mathbf{u}(t)+G(t) \mathbf{w}(t) \\
\mathbf{z}(t)=H \mathbf{x}(t)+\mathbf{v}(t)
\end{gathered}
$$

In assuming a constant sampling interval $\Delta t$ in the gyro output, the system equation Eq. (24) and observation equations Eq. (25) can be discretized and rewritten as

$$
\begin{gathered}
\mathbf{x}_{k+1}=\Phi_{k} \mathbf{x}_{k}+\Gamma_{k} \mathbf{u}_{k}+\Upsilon_{k} \mathbf{w}_{k} \\
\mathbf{z}_{k}=H_{k} \mathbf{x}_{k}+\mathbf{v}_{k}
\end{gathered}
$$

where

$$
\Phi_{k}=e^{\mathbf{A} \Delta t}=\left[\begin{array}{cc}
1 & -\Delta t \\
0 & 1
\end{array}\right]
$$

and

$$
\Gamma_{k}=\int_{0}^{\Delta t} e^{A \tau} B d \tau=\left[\begin{array}{c}
\Delta t \\
0
\end{array}\right]
$$

The process noise covariance matrix used in the propagation of the estimation error covariance given by (Gelb, 1974; Crassidis \& Junkins, 2004)

$$
\left(\Upsilon_{k} Q_{k} \Upsilon_{k}\right)^{T}=\int_{t_{k}}^{t_{k+1}} \int_{t_{k}}^{t_{k+1}} \Phi\left(t_{k+1}, \tau\right) G(\tau) E\left\{\mathbf{w}(\tau) \mathbf{w}^{T}(\alpha)\right\} G^{T}(\alpha) \Phi\left(t_{k+1}, \alpha\right) d \tau d \alpha
$$

can be properly numerically estimated given a sufficiently small sampling interval by following the numerical solution by van Loan (Crassidis \& Junkins, 2004). First, the following $2 n \times 2 n$ matrix is formed:

$$
\boldsymbol{a}=\left[\begin{array}{cc}
-A & G Q G^{T} \\
0 & A^{T}
\end{array}\right] \Delta t
$$

where $\Delta t$ is the constant sampling interval, $A$ and $G$ are the constant continuous-time state matrix and error distribution matrix given in Eq. (24), and $Q$ is the constant continuoustime process noise covariance matrix 


$$
Q=E\left\{\mathbf{w}(t) \mathbf{w}^{T}(t)\right\}=\left[\begin{array}{cc}
\sigma_{g}^{2} & 0 \\
0 & \sigma_{\beta g}^{2}
\end{array}\right]
$$

The matrix exponential of Eq. (31) is then computed by

$$
\mathscr{B}=e^{a} \equiv\left[\begin{array}{cc}
\mathscr{B}_{11} & \boldsymbol{B}_{12} \\
0 & \mathscr{B}_{22}
\end{array}\right]=\left[\begin{array}{cc}
\mathscr{B}_{11} & \Phi_{k}^{-1} \mathcal{Q}_{k} \\
0 & \Phi_{k}^{T}
\end{array}\right]
$$

where $\Phi_{k}$ is the state transition matrix from Eq. (28) and $\mathscr{Q}_{k}=\left(\Upsilon_{k} Q_{k} \Upsilon_{k}\right)^{T}$. Therefore, the discrete-time process noise covariance is

$$
\mathcal{Q}_{k}=\left(\Upsilon_{k} Q_{k} \Upsilon_{k}\right)^{T}=\Phi_{k} \boldsymbol{B}_{12}=\left[\begin{array}{cc}
1 / 3 \sigma_{\beta g}^{2} \Delta t^{3}+\sigma_{g}^{2} \Delta t & -1 / 2 \sigma_{\beta g}^{2} \Delta t^{2} \\
-1 / 2 \sigma_{\beta g}^{2} \Delta t^{2} & \sigma_{\beta g}^{2} \Delta t
\end{array}\right]
$$

The discrete-time measurement noise covariance is

$$
r_{k}=E\left\{\mathbf{v}_{k} \mathbf{v}_{k}^{T}\right\}=\sigma_{\theta m}^{2}
$$

Given the filter model as expressed in Eqs. (22) and (23), the estimated states and error covariance are initialized where this initial error covariance is given by $P_{0}=E\left\{\tilde{\mathbf{x}}\left(t_{0}\right) \tilde{\mathbf{x}}^{T}\left(t_{0}\right)\right\}$. If a measurement is given at the initial time, then the state and covariance are updated using the Kalman gain formula

$$
K_{k}=P_{k}^{-} H_{k}^{T}\left[H_{k} P_{k}^{-} H_{k}^{T}+r_{k}\right]^{-1}
$$

where $P_{k}^{-}$is the a priori error covariance matrix and is equal to $P_{0}$. The updated or $a$ posteriori estimates are determined by

$$
\begin{aligned}
\hat{\mathbf{x}}_{k}^{+} & =\hat{\mathbf{x}}_{k}^{-}+K_{k}\left[z_{k}-H_{k} \hat{\mathbf{x}}_{k}^{-}\right] \\
P_{k}^{+} & =\left[I_{2 x 2}-K_{k} H_{k}\right] P_{k}^{-}
\end{aligned}
$$

where again with a measurement given at the initial time, the a priori state $\hat{\mathbf{x}}_{k}^{-}$is equal to $\hat{\mathbf{x}}_{0}$. The state estimate and covariance are propagated to the next time step using

$$
\begin{aligned}
\hat{\mathbf{x}}_{k+1}^{-} & =\Phi_{k} \hat{\mathbf{x}}_{k}^{+}+\Gamma_{k} u_{k} \\
P_{k+1}^{-} & =\Phi_{k} P_{k}^{+} \Phi_{k}^{T}+\mathcal{Q}_{k}
\end{aligned}
$$


If a measurement isn't given at the initial time step or any time step during the process, the estimate and covariance are propagated to the next available measurement point using Eq. (38).

\subsubsection{Translation Linear Quadratic Estimator}

With the measured translation state from the iGPS sensor, being given by

$$
\mathbf{z}=\underbrace{\left[\begin{array}{llll}
1 & 0 & 0 & 0 \\
0 & 1 & 0 & 0
\end{array}\right]}_{C} \underbrace{\left[X, Y, V_{x}, V_{y}\right]^{T}}_{\mathbf{x}}
$$

the dynamics of a full-order state estimator is described by the equation

$$
\begin{aligned}
\dot{\tilde{\mathbf{x}}} & =\dot{\mathbf{x}}-\dot{\hat{\mathbf{x}}}=[A \mathbf{x}+B \mathbf{u}]-\left[A \hat{\mathbf{x}}+B \mathbf{u}+L_{L Q E}(\mathbf{z}-C \hat{\mathbf{x}})\right] \\
& =A(\mathbf{x}-\hat{\mathbf{x}})-L_{L Q E}(C \mathbf{x}-C \hat{\mathbf{x}}) \\
& =\left(A-L_{L Q E} C\right) \tilde{\mathbf{x}}
\end{aligned}
$$

where

$$
\begin{array}{rrl}
A \mathbf{x}+B \mathbf{u} & : & \text { linearized plant dynamics } \\
A \hat{\mathbf{x}}+B \mathbf{u} & : & \text { system model } \\
L_{L Q E} & : & \text { linear quadratic estimator gain matrix } \\
C \hat{\mathbf{x}} \quad: & \text { measurement if } \mathbf{x} \text { were } \hat{\mathbf{x}}
\end{array}
$$

The observer gain matrix $L_{L Q E}$ can be solved using standard linear quadratic estimator methods as (Bryson, 1993)

$$
L_{L Q E}=P C^{T} R_{T}^{-1}
$$

where $P$ is the solution to the algebraic Riccati equation

$$
A P+P A^{T}-P C^{T} R_{T}^{-1} C P+Q_{T}=0
$$

and $Q_{T}$ and $R_{T}$ are the associated weighting matrices with respect to the translational degree of freedom defined as

$$
\begin{aligned}
& Q_{T}=\operatorname{diag}\left(1 /\left\|\Delta X_{\max }\right\|^{2}, 1 /\left\|\Delta Y_{\max }\right\|^{2}, 1 /\left\|\Delta V_{y, \max }\right\|^{2}, 1 /\left\|\Delta V_{x, \max }\right\|^{2}\right) \\
& R_{T}=\operatorname{diag}\left(1 /\left(F_{\max }\right)^{2}, 1 /\left(F_{\max }\right)^{2}\right)
\end{aligned}
$$

where $\Delta X_{\max }, \Delta Y_{\max }, \Delta V_{x, \max }, \Delta V_{y, \max }$ are taken to be the maximum allowed errors between the current and estimated translational states and $F_{\max }$ is the maximum possible imparted force from the thrusters.

Table 3 lists the values of the attitude Kalman filter and translation state observer used for the experimental tests. 


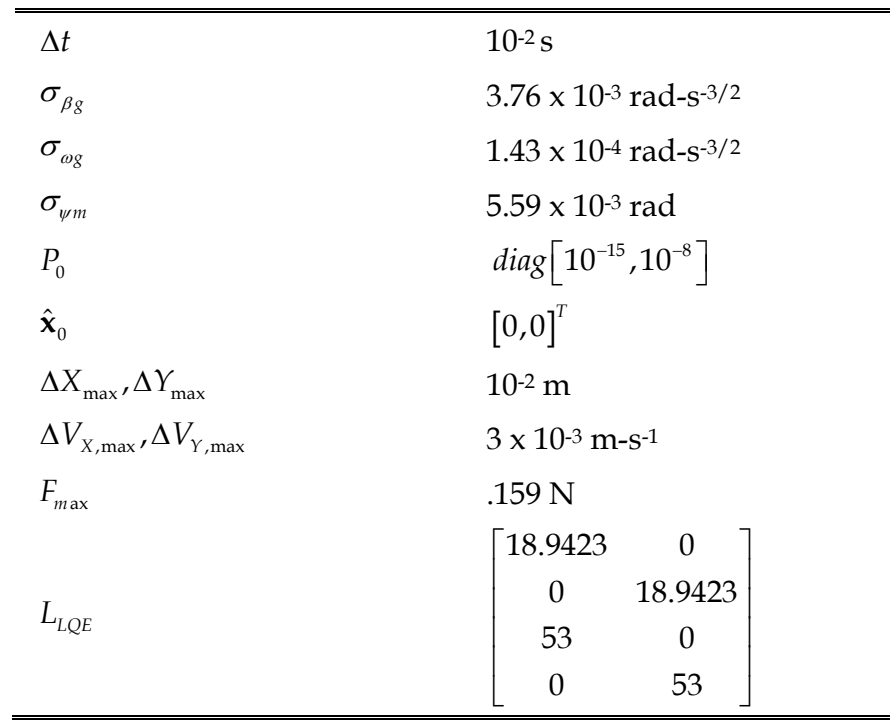

Table 3. Kalman Filter Estimation Paramaters

\subsection{Smooth Feedback Control via State Feedback Linearization and Linear Quadratic Regulation}

Considering a Multi-Input Multi-Output (MIMO) nonlinear system in control-affine form, the state feedback linearization problem of nonlinear systems can be stated as follows: obtain a proper state transformation

$$
\mathbf{z}=\Phi(\mathbf{x}) \text { where } \mathbf{z} \in \mathbb{R}^{N_{x}}
$$

and a static feedback control law

$$
\mathbf{u}=\alpha(\mathbf{x})+\beta(\mathbf{x}) \mathbf{v} \text { where } \mathbf{v} \in \mathbb{R}^{N_{u}}
$$

such that the closed-loop system in the new coordinates and controls become

$$
\dot{\mathbf{z}}=\left.\left\{\frac{\partial \Phi}{\partial \mathbf{x}}[\mathbf{f}(\mathbf{x})+G(\mathbf{x}) \alpha(\mathbf{x})]\right\}\right|_{\mathbf{x}=\Phi^{-1}(\mathbf{z})}+\left.\left\{\frac{\partial \Phi}{\partial \mathbf{x}}[G(\mathbf{x}) \boldsymbol{\beta}(\mathbf{x})]\right\}\right|_{\mathbf{x}=\Phi^{-1}(\mathbf{z})} \mathbf{v}
$$

is both linear and controllable. The necessary conditions for a MIMO system to be considered for input-output linearization are that the system must be square or $N_{u}=N_{y}$ where $N_{u}$ is defined as above to be the number of control inputs and $N_{y}$ is the number of outputs for a system of the expanded form (Isidori, 1989; Slotine, 1990)

$$
\begin{aligned}
& \dot{\mathbf{x}}=\mathbf{f}(\mathbf{x})+G(\mathbf{x}) \mathbf{u} \\
& \mathbf{y}=\sum_{i=1}^{N_{y}} h_{i}(\mathbf{x})=\mathbf{h}(\mathbf{x})
\end{aligned}
$$


The input-output linearization is determined by differentiating the outputs $y_{i}$ in Eq. (47) until the inputs appear. Following the method outlined in (Slotine, 1990) by which the assumption is made that the partial relative degree $r_{i}$ is the smallest integer such that at least one of the inputs appears in $y_{i}^{\left(r_{i}\right)}$, then

$$
y_{i}^{\left(r_{i}\right)}=L_{\mathbf{f}}^{r_{i}} h_{i}+\sum_{j=1}^{N_{y}} L_{\mathbf{g}_{j}} L_{\mathbf{f}}^{r_{i}-1} h_{i}(\mathbf{x}) u_{j}
$$

with the restriction that $L_{\mathbf{g}_{j}} L_{\mathbf{f}}^{r_{i}-1} h_{i}(\mathbf{x}) \neq 0$ for at least one $j$ in a neighborhood of the equilibrium point $\mathbf{x}_{0}$. Letting

$$
E(\mathbf{x})=\left[\begin{array}{ccc}
L_{\mathbf{g}_{1}} L_{\mathbf{f}}{ }^{r_{1}-1} h_{1}(\mathbf{x}) & \cdots & L_{\mathbf{g}_{N_{u}}} L_{\mathbf{f}}{ }^{r_{1}-1} h_{1}(\mathbf{x}) \\
L_{\mathbf{g} 1} L_{\mathbf{f}}{ }^{r_{2}-1} h_{2}(\mathbf{x}) & \cdots & L_{\mathbf{g}_{N_{u}}} L_{\mathbf{f}}{ }^{r_{2}-1} h_{2}(\mathbf{x}) \\
\cdots & \cdots & \cdots \\
L_{\mathbf{g}_{1}} L_{\mathbf{f}}{ }^{r_{N_{y}}-1} h_{N_{y}}(\mathbf{x}) & \cdots & L_{\mathbf{g}_{N_{u}}} L_{\mathbf{f}}{ }^{r_{N_{y}}-1} h_{N_{y}}(\mathbf{x})
\end{array}\right]
$$

so that Eq. (49) is in the form

$$
\left[\begin{array}{c}
y_{1}{ }^{\left(r_{1}\right)} \\
y_{2}{ }^{\left(r_{2}\right)} \\
\cdots \\
y_{N_{y}}{ }^{\left({ }_{N_{y}}\right)}
\end{array}\right]=\left[\begin{array}{c}
L_{\mathbf{f}}^{{ }^{r_{1}}} h_{1}(\mathbf{x}) \\
L_{\mathbf{f}}{ }^{r_{2}} h_{2}(\mathbf{x}) \\
\cdots \\
L_{\mathrm{f}}{ }^{r_{N_{y}}} h_{N_{y}}(\mathbf{x})
\end{array}\right]+E(\mathbf{x}) \mathbf{u}
$$

the decoupling control law can be found where the $N_{y} \times N_{y}$ matrix $E(\mathbf{x})$ is invertible over the finite neighborhood of the equilibrium point for the system as

$$
\mathbf{u}=E(\mathbf{x})^{-1}\left[\begin{array}{c}
v_{1}-L_{\mathbf{f}}{ }^{r_{1}} h_{1}(\mathbf{x}) \\
v_{2}-L_{\mathbf{f}}{ }^{r_{2}} h_{2}(\mathbf{x}) \\
\cdots \\
v_{N_{y}}-L_{\mathbf{f}}^{{ }^{r_{N y}}} h_{N_{y}}(\mathbf{x})
\end{array}\right]
$$

With the above stated equations for the simulator dynamics in Eq. (9) given $G_{1}(\mathbf{x})$ as defined in Eq. (11), if we choose

$$
\mathbf{h}(\mathbf{x})=[X, Y, \psi]^{T}
$$

the state transformation can be chosen as

$$
\mathbf{z}^{T}=\left[h_{1}(\mathbf{x}), h_{2}(\mathbf{x}), h_{3}(\mathbf{x}), L_{\mathrm{f}} h_{1}(\mathbf{x}), L_{\mathrm{f}} h_{2}(\mathbf{x}), L_{\mathrm{f}} h_{3}(\mathbf{x})\right]=\left[X, Y, \psi, V_{x}, V_{y}, \omega_{z}\right]
$$


where $\mathbf{z}^{T}=\left[z_{1}, z_{2}, \ldots, z_{6}\right] \in \mathbb{R}^{6}$ are new state variables, and the system in Eq. (9) is transformed into

$$
\dot{\mathbf{z}}^{T}=\left[z_{4}, z_{5}, z_{6}, m^{-1}\left(\mathrm{c} z_{3}{ }^{B} F_{x}-\mathrm{s} z_{3}{ }^{B} F_{y}\right), m^{-1}\left(\mathrm{~s} z_{3}{ }^{B} F_{x}+\mathrm{c} z_{3}{ }^{B} F_{y}\right), J_{z}^{-1 B} T\right]
$$

The dynamics given by Eq. (9) considering the switching logic described in Eqs. (10), (12) and (14) can now be transformed using Eq. (54) and the state feedback control law

$$
\left[{ }^{B} \mathbf{F},{ }^{B} T\right]=E(\mathbf{x})^{-1}(\mathbf{v}-\mathbf{b})
$$

into a linear system

$$
\dot{\mathbf{Z}}=\left[\begin{array}{ll}
\mathbf{0}_{3 \times 3} & \mathbf{I}_{3 \times 3} \\
\mathbf{0}_{3 \times 3} & \mathbf{0}_{3 \times 3}
\end{array}\right] \mathbf{z}+\left[\begin{array}{l}
\mathbf{0}_{3 \times 3} \\
\mathbf{I}_{3 \times 3}
\end{array}\right] \mathbf{v}
$$

where

$$
\mathbf{b}=\left[L_{\mathrm{f}}^{r_{1}} h_{1}(\mathbf{x}), L_{\mathrm{f}}^{r_{2}} h_{2}(\mathbf{x}), L_{\mathrm{f}}^{r_{3}} h_{3}(\mathbf{x})\right]^{T}
$$

and $E(\mathbf{x})$ given by Eq. (49) with equivalent inputs $\mathbf{v}=\left[v_{1}, v_{2}, v_{3}\right]^{T}$ and relative degree of the system at the equilibrium point $\mathbf{x}_{0}$ is $\left(r_{1}, r_{2}, r_{3}\right)=(2,2,2)$. Therefore the total relative degree of the system at the equilibrium point, which is defined as the sum of the relative degree of the system, is six. Given that the total relative degree of the system is equal to the number of states, the nonlinear system can be exactly linearized by state feedback and with the equivalent inputs $v_{i}$, both stabilization and tracking can be achieved for the system without concern for the stability of the internal dynamics (Slotine, 1990).

One of the noted limitations of a feedback linearized based control system is the reliance on a fully measured state vector (Slotine, 1990). This limitation can be overcome through the employment of proper state estimation. HIL experimentation on SRL's second generation robotic spacecraft simulator using these navigation algorithms combined with the state feedback linearized controller as described above coupled with a linear quadratic regulator to ensure the poles of Eq. (56) lie in the open left half plane demonstrate satisfactory results as reported in the following section.

\subsubsection{Feedback Linearized Control Law with MSGCMG Rotational Control and Thruster Translational Control}

By applying Eq. (55) to the dynamics in Eq. (9) given $G_{1}(\mathbf{x})$ as defined in Eq. (11) where the system is taken to be observable in the state vector $\mathbf{y}=[X, Y, \psi]^{T}=\left[x_{1}, x_{2}, x_{3}\right]^{T}$ and by using thruster two for translational control (i.e. for the case ${ }^{B} U_{x}>0$ where ${ }^{B} U_{x}=v_{1} \mathrm{c} \psi+v_{2} \mathrm{~s} \psi$ and $\left.{ }^{B} U_{y}=-v_{1} \mathrm{~s} \psi+v_{2} \mathrm{c} \psi\right)$, the feedback linearized control law is

$$
\mathbf{u}^{T}=\left[{ }^{B} F_{x},{ }^{B} F_{y}{ }^{B} T_{z}\right]=\left[m^{B} U_{x}, m^{B} U_{x}, m L^{B} U_{y}+J_{z} v_{3}\right]
$$


which is valid for all $\mathbf{x}$ in a neighborhood of the equilibrium point $\mathbf{x}_{0}$. Similarly, the feedback linearized control law when ${ }^{B} U_{x}<0$ (thruster one is providing translation control)

$$
\mathbf{u}^{T}=\left[{ }^{B} F_{x},{ }^{B} F_{y},{ }^{B} T_{z}\right]=\left[m^{B} U_{x}, m^{B} U_{y},-m L^{B} U_{y}+J_{z} v_{3}\right]
$$

Finally, when ${ }^{B} U_{x}=0$ (both thrusters used for translational control) given $G_{1}(\mathbf{x})$ as defined in Eq. (13) is

$$
\mathbf{u}^{T}=\left[{ }^{B} F_{y},{ }^{B} T_{z}\right]=\left[m^{B} U_{y} / 2, J_{z} v_{3}\right]
$$

\subsubsection{Feedback Linearized Control Law for Thruster Roto-Translational Control}

As mentioned previously, by considering a momentum exchange device for rotational control, momentum storage must be managed. For a control moment gyroscope based moment exchange device, desaturation is necessary near gimbal angles of $\pi / 2$. In this region, due to the mathematical singularity that exists, very little torque can be exchanged with the vehicle and thus it is essentially ineffective as an actuator. To accommodate these regions of desaturation, logic can be easily employed to define controller modes as follows: If the MSGCMG is being used as a control input and if the gimbal angle of the MSGCMG is greater than 75 degrees, the controller mode is switched from normal operation mode to desaturation mode and the gimbal angle rate is directly commanded to bring the gimbal angle to a zero degree nominal position while the thruster not being directly used for translational control is slewed as appropriate to provide torque compensation. In these situations, the feedback linearizing control law for the system dynamics in Eq. (9) given $G_{1}(\mathbf{x})$ as defined in Eq. (15) where thruster two is providing translational control $\left({ }^{B} U_{x}>0\right)$, and thruster one is providing the requisite torque is

$$
\mathbf{u}^{T}=\left[{ }^{B} F_{x},{ }^{B} F_{y}, T_{z}\right]=\left[m^{B} U_{x},\left(m L^{B} U_{y}-J_{z} v_{3}\right) / 2 L,\left(m L^{B} U_{y}+J_{z} v_{3}\right) / 2\right]
$$

Similarly, the feedback linearizing control law for the system assuming thruster one is providing translational control $\left({ }^{B} U_{x} \leq 0\right)$ while thruster two provides the requisite torque is

$$
\mathbf{u}^{T}=\left[{ }^{B} F_{x},{ }^{B} F_{y}, T_{z}\right]=\left[m^{B} U_{x},\left(m L^{B} U_{y}+J_{z} v_{3}\right) / 2 L,\left(m L^{B} U_{y}-J_{z} v_{3}\right) / 2\right]
$$

\subsubsection{Determination of the thruster angles, forces and MSGCMG gimbal rates}

In either mode of operation, the pertinent decoupling control laws are used to determine the commanded angle for the thrusters and whether or not to open or close the solenoid for the thruster. For example, if ${ }^{B} U_{x}>0$, Eq. (58) or (61) can be used to determine the angle to command thruster two as

$$
\alpha_{2}=\tan ^{-1}\left({ }^{B} F_{y} /{ }^{B} F_{x}\right)
$$

and the requisite thrust as 


$$
F_{2}=\sqrt{{ }^{B} F_{x}^{2}+{ }^{B} F_{y}^{2}}
$$

If the MSGCMG is being used, the requisite torque commanded to the CMG is taken directly from Eq. (58). In the normal operation mode, with the commanded angle for thruster one not pertinent, it can be commanded to zero without affecting control of the system. Similarly, if ${ }^{B} U_{X}<0$, Eq. (59) or (62) can be used to determine the angle to command thruster one and the requisite thrust analogous to Eqs. (63) and (64). The requisite torque commanded to the CMG is similarly taken directly from Eq. (59). The required CMG torques can be used to determine the gimbal rate $\left(\dot{\delta}_{C M G}\right)$ to command the MSGCMG by solving the equation (Hall, 2006; Romano \& Hall, 2006)

$$
\dot{\delta}_{C M G}=-T_{C M G} /\left(h_{w} \cos \delta_{C M G}\right)
$$

where $h_{w}$ is the constant angular momentum of the rotor wheel and $\delta_{C M G}$ is the current angular displacement of the wheel's rotational axis with respect to the horizontal.

If the momentum exchange device is no longer available and ${ }^{B} U_{x}>0$, the thruster angle commands and required thrust value for the opposing thruster can be determined by using Eq. (61) as

$$
\alpha_{1}=-\pi / 2 \operatorname{sign}\left(m L^{B} U_{y}+J_{z} v_{3}\right)
$$

and

$$
F_{1}=-\operatorname{sign}\left(\alpha_{1}\right)\left(m L^{B} U_{y}+J_{z} v_{3}\right) / L
$$

given ${ }^{B} T_{z}=-F_{1} \mathrm{~s} \alpha_{1} L$. Likewise, the thruster angle commands and required thrust value for the opposing thruster given ${ }^{B} U_{x}<0$ can be determined by using Eq. (62) as

$$
\alpha_{2}=\pi / 2 \operatorname{sign}\left(m L^{B} U_{y}-J_{z} v_{3}\right)
$$

and

$$
F_{2}=\operatorname{sign}\left(\alpha_{2}\right)\left(m L^{B} U_{y}-J_{z} v_{3}\right) / L
$$

given ${ }^{B} T_{z}=-F_{2} \sin \alpha_{2} L$.

\subsubsection{Linear Quadratic Regulator Design}

In order to determine the linear feedback gains used to compute the requisite equivalent inputs $v_{i}$ to regulate the three degrees of freedom so that

$$
\begin{array}{lll}
\lim _{t \rightarrow \infty} z_{1}(t)=X(t)=X_{\text {ref }}, & \lim _{t \rightarrow \infty} z_{2}(t)=Y(t)=Y_{\text {ref }}, & \lim _{t \rightarrow \infty} z_{3}(t)=\psi(t)=\psi_{\text {ref }} \\
\lim _{t \rightarrow \infty} z_{4}(t)=V_{X}(t)=V_{X, \text { ref }}, & \lim _{t \rightarrow \infty} z_{5}(t)=V_{Y}(t)=V_{Y, \text { ref }}, & \lim _{t \rightarrow \infty} z_{6}(t)=\omega_{z}(t)=\omega_{z, \text { ref }}
\end{array}
$$


a standard linear quadratic regulator is employed where the state-feedback law $\mathbf{v}=-K \mathbf{z}$ minimizes the quadratic cost function

$$
J(\mathbf{v})=\int_{0}^{\infty}\left(\mathbf{z}^{T} Q \mathbf{z}+\mathbf{v}^{T} R \mathbf{v}\right) d t
$$

subject to the feedback linearized state-dynamics of the system given in Eq. (56). Given the relation between the linearized state and true state of the system, the corresponding gain matrices $R$ and $Q$ in Eq. (71) are chosen to minimize the appropriate control and state errors as

$$
\begin{aligned}
& Q=\operatorname{diag}\left(\begin{array}{l}
1 /\left\|\Delta X_{\max }\right\|^{2},, 1 /\left\|\Delta Y_{\max }\right\|^{2}, 1 /\left\|\Delta \psi_{\max }\right\|^{2}, \\
1 /\left\|\Delta V_{y, \max }\right\|^{2}, 1 /\left\|\Delta V_{x, \max }\right\|^{2}, 1 /\left\|\Delta \omega_{z, \max }\right\|^{2}
\end{array}\right) \\
& R=\operatorname{diag}\left(1 /\left(F_{\max }\right)^{2}, 1 /\left(F_{\max }\right)^{2}, 1 /\left(T_{C M G, \max }\right)^{2}\right)
\end{aligned}
$$

where $\Delta X_{\max }, \Delta Y_{\max }, \Delta V_{x, \max }, \Delta V_{y, \max }, \Delta \psi_{\max }, \Delta \omega_{z, \max }$ are taken to be the maximum errors allowed between the current states and reference states while $F_{\max }$ and $T_{C M G \text {, max }}$ are taken to be the maximum possible imparted force and torques from the thrusters and MSGCMG respectively.

Given the use of discrete cold-gas thrusters in the system for translational control throughout a commanded maneuver and rotational control when the continuously acting momentum exchange device is unavailable, Schmitt trigger switching logic is imposed. Schmitt triggers have the unique advantage of reducing undesirable chattering and subsequent propellant waste nearby the reference state through an output-versus-input logic that imposes a dead zone and hysteresis to the phase space as shown in Fig. $\mathbf{5}$.
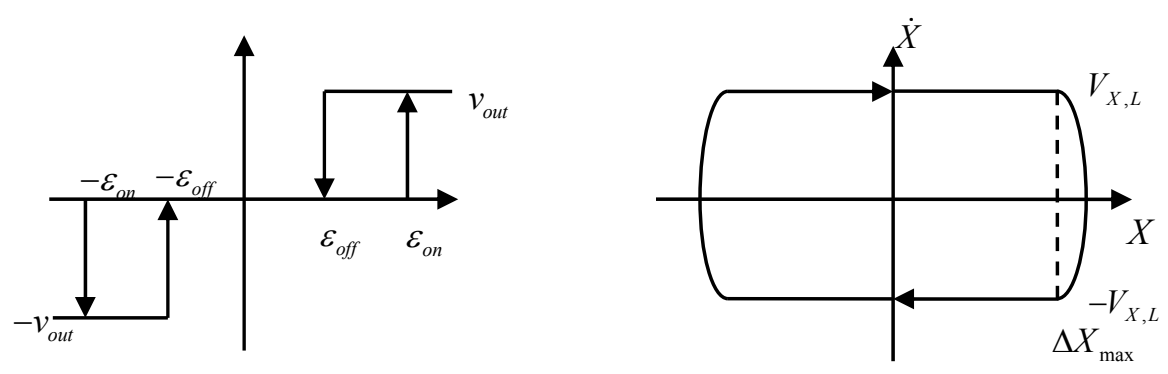

Fig. 5. Schmitt Trigger Characteristics with Design Parameters Considering X Coordinate Control Logic

Three separate Schmitt triggers are used with the design parameters of the Schmitt trigger shown in Fig. 5 (as demonstrated for the X coordinate control logic). In the case of the two translational DoF Schmitt triggers, the parameters are chosen such that 


$$
\begin{aligned}
& \varepsilon_{o n}=K_{X} X_{d b}+K_{\dot{X}} V_{X, L} \\
& \varepsilon_{o f f}=K_{X} X_{d b}-K_{\dot{X}} V_{X, L}
\end{aligned}
$$

where $V_{X, L}=V_{Y, L}=\dot{X}_{L}=F_{\max } \Delta t / 2 m . X_{d b}, Y_{d b}$ are free parameters that are constrained by mission requirements. $v_{\text {out }}$ is chosen such that the maximum control command from the decoupling control law yields a value less than or equal to $F_{\max }$ for the translational thruster. In the case of the rotational DoF Schmitt trigger when the momentum exchange device is unavailable, the parameters are chosen such that

$$
\begin{aligned}
& \varepsilon_{\text {on }}=K_{\psi} \psi_{d b}+K_{\omega_{z}} \omega_{z, L} \\
& \varepsilon_{\text {off }}=K_{\psi} \psi_{d b}-K_{\omega_{z}} \omega_{z, L}
\end{aligned}
$$

where $\omega_{z, L}=F_{\max } L \Delta t / 2 J_{z} \cdot \psi_{d b}$ is a free parameter that is again constrained by mission requirements. For both modes of operation (i.e. with or without a momentum exchange device), Eqs. (58) through (60) can be used to determine that

$$
v_{1, \max }=v_{2, \max }=F_{\max } / \sqrt{2} m
$$

and when the thrusters are used for rotational control

$$
v_{3, \max }=F_{\max } L / J_{z}
$$

When the momentum exchange device is available, the desired torque as determined by the LQR control law as described above is passed directly through the Schmitt trigger to the decoupling control law to determine the required gimbal rate command to the MSGCMG. The three Schmitt trigger blocks output the requested control inputs along the ICS frame. The appropriate feedback linearizing control law is then used to transform these control inputs into requested thrust, thruster angle and MSGCMG gimbal rate along the BCS frame. From these, a vector of specific actuator commands are formed such that

$$
\mathbf{u}_{c}^{T}=\left[F_{1}, \alpha_{1}, F_{2}, \alpha_{2}, \dot{\delta}_{\mathrm{CMG}}\right]
$$

Each thruster command is normalized with respect to $F_{\max }$ and then fed with its corresponding commanded angle into separate Pulse Width Modulation (PWM) blocks. Each PWM block is then used to obtain an approximately linear duty cycle from on-off actuators by modulating the opening time of the solenoid valves (Wie, 1998). Additionally, due to the linkage between the thruster command and the thruster angle, the thruster firing sequence is held until the actual thruster angle is within a tolerance of the commanded thruster angle. Furthermore, in order to reduce over-controlling the system, the LQR, Schmitt trigger logic and decoupling control algorithm are run at the PWM bandwidth of $8.33 \mathrm{~Hz}$. From each PWM, digital outputs (either zero or one) command the two thrusters while the corresponding angle is sent via RS-232 to the appropriate thruster gimbal motor. 


\begin{tabular}{|c|c|}
\hline$\Delta t$ & $10^{-2} \mathrm{~s}$ \\
\hline$\Delta X_{\max }, \Delta Y_{\max }$ & $10^{-2} \mathrm{~m}$ \\
\hline$\Delta V_{X, \max }, \Delta V_{Y, \max }$ & $3 \times 10^{-3} \mathrm{~m}-\mathrm{s}^{-1}$ \\
\hline$\Delta \psi_{\max }$ & $1.8 \times 10^{-2} \mathrm{rad}$ \\
\hline$\Delta \omega_{z, \max }$ & $1.8 \times 10^{-2} \mathrm{rad}^{-\mathrm{s}^{-1}}$ \\
\hline$F_{\max }$ & $.159 \mathrm{~N}$ \\
\hline$T_{C M G, \max }$ & $.668 \mathrm{Nm}$ \\
\hline$K_{X}=K_{Y}=K_{L Q R}(1,1)=K_{L Q R}(2,2)$ & 15.9 \\
\hline$K_{\dot{X}}=K_{\dot{Y}}=K_{L Q R}(1,4)=K_{L Q R}(2,5)$ & $84.54 \mathrm{~s}$ \\
\hline$K_{\psi}=K_{L Q R}(3,3)$ & 1.39 \\
\hline$K_{\omega_{z}}=K_{L Q R}(3,6)$ & $1.75 \mathrm{~s}$ \\
\hline$X_{d b}=Y_{d b}$ & $10^{-2} \mathrm{~m}$ \\
\hline$V_{X, L}=V_{Y, L}$ & $3.05 \times 10^{-5} \mathrm{~m}-\mathrm{s}^{-1}$ \\
\hline$\psi_{d b}$ & $1.8 \times 10^{-2} \mathrm{rad}$ \\
\hline$\omega_{z, L}$ & $1.8 \times 10^{-2} \mathrm{rad}^{-\mathrm{s}^{-1}}$ \\
\hline$\varepsilon_{o n}(X)=\varepsilon_{o n}(Y)$ & $1.61 \times 10^{-1} \mathrm{~m}$ \\
\hline$\varepsilon_{o f f}(X)=\varepsilon_{o f f}(Y)$ & $1.56 \times 10^{-1} \mathrm{~m}$ \\
\hline$\varepsilon_{o n}(\psi)$ & $2.47 \times 10^{-2} \mathrm{rad}$ \\
\hline$\varepsilon_{\text {off }}(\psi)$ & $2.37 \times 10^{-2} \mathrm{rad}$ \\
\hline PWM min pulse width & $10^{-2} \mathrm{~s}$ \\
\hline PWM sample time & $1.2 \times 10^{-1} \mathrm{~s}$ \\
\hline
\end{tabular}

Table 4. Values of the Control Parameters

Table 4 lists the values of the control parameters used for the experimental tests reported in the following section. In particular, $\Delta V_{X, \max }, \Delta V_{Y, \max }$ are chosen based typical maximum relative velocities during rendezvous scenarios while $\Delta \psi_{\max }$ is taken to be 1 degree and $\Delta \omega_{z \text {,max }}$ is chosen to be 1 degrees/sec which correspond to typical slew rate requirements for small satellites (Roser \& Schedoni, 1997;Lappas et al., 2002). The minimum opening time of the PWM was based on experimental results for the installed solenoid valves reported in (Lugini \& Romano, 2009).

\section{Experimental Results}

The navigation and control algorithms introduced above were coded in MATLAB®Simulink ${ }^{\circledR}$ and run in real time using MATLAB XPC Target ${ }^{\mathrm{TM}}$ embedded on the SRL's second generation spacecraft simulator's on-board PC-104. Two experimental tests are 
presented to demonstrate the effectiveness of the designed control system. The scenario presented represents a potential real-world autonomous proximity operation mission where a small spacecraft is tasked with performing a full 360 degree circle around another spacecraft for the purpose of inspection or pre-docking. These experimental tests validate the navigation and control approach and furthermore demonstrate the capability of the robotic spacecraft simulator testbed.

\subsection{Autonomous Proximity Maneuver using Vectorable Thrusters and MSGCMG along a Closed Circular Path}

Fig. 6, Fig. 7, and Fig. 8 report the results of an autonomous proximity maneuver along a closed circular trajectory of NPS SRL's second generation robotic spacecraft simulator using its vectorable thrusters and MSGCMG. The reference path for the center of mass of the simulator consists of 200 waypoints, taken at angular intervals of 1.8 deg along a circle of diameter $1 \mathrm{~m}$ with a center at the point $[2.0 \mathrm{~m}, 2.0 \mathrm{~m}]$ in the ICS, which can be assumed, for instance, to be the center of mass of the target. The reference attitude is taken to be zero throughout the maneuver. The entire maneuver lasts $147 \mathrm{~s}$. During the first $10 \mathrm{~s}$, the simulator is maintained fixed in order to allow the attitude Kalman filter time to converge to a solution. At $10 \mathrm{~s}$ into the experiment, the solenoid valve regulating the air flow to the linear air bearings is opened and the simulator begins to float over the epoxy floor. At this point, the simulator begins to follow the closed path through autonomous control of the two thrusters and the MSGCMG.

As evidenced in Fig. 6a through Fig. 6d, the components of the center of mass of the simulator as estimated by the translation linear quadratic estimator are kept close to the reference signals by the action of the vectorable thrusters. Specifically, the mean of the absolute value of the tracking error is $1.3 \mathrm{~cm}$ for $\Delta X$, with a standard deviation of $9.1 \mathrm{~mm}$, $1.4 \mathrm{~cm}$ mean for $\Delta Y$ with a standard deviation of $8.6 \mathrm{~mm}, 2.4 \mathrm{~mm} / \mathrm{s}$ mean for $\Delta V_{X}$ with a standard deviation of $1.8 \mathrm{~mm} / \mathrm{s}$ and $3.0 \mathrm{~mm} / \mathrm{s}$ mean for $\Delta V_{Y}$ with a standard deviation of $2.7 \mathrm{~mm} / \mathrm{s}$. Furthermore, the mean of the absolute value of the estimated error in $X$ is $2 \mathrm{~mm}$ with a standard deviation of $2 \mathrm{~mm}$ and $4 \mathrm{~mm}$ in $Y$ with a standard deviation of $3 \mathrm{~mm}$. Likewise, Fig. 6e and Fig. 6f demonstrate the accuracy of the attitude tracking control through a comparison of the commanded and actual attitude and attitude rate. Specifically, the mean of the absolute value of tracking error for $\Delta \psi$ is 0.14 deg with a standard deviation of $0.11 \mathrm{deg}$ and $0.14 \mathrm{deg} / \mathrm{s}$ for $\Delta \omega_{z}$ with a standard deviation of $0.15 \mathrm{deg} / \mathrm{s}$. These control accuracies are in good agreement with the set parameters of the Schmitt triggers and the LQR design.

Fig. 7a through Fig. 7d report the command signals to the simulator's thrusters along with their angular positions. The commands to the thrusters demonstrate that the Schmitt trigger logic successfully avoids chattering behavior and the feedback linearized controller is able to determine the requisite thruster angles. Fig. 7e and Fig. $7 \mathrm{f}$ show the gimbal position of the miniature single-gimbaled control moment gyro and the delivered torque. Of note, the control system is able to autonomously maneuver the simulator without saturating the MSGCMG. 
a)
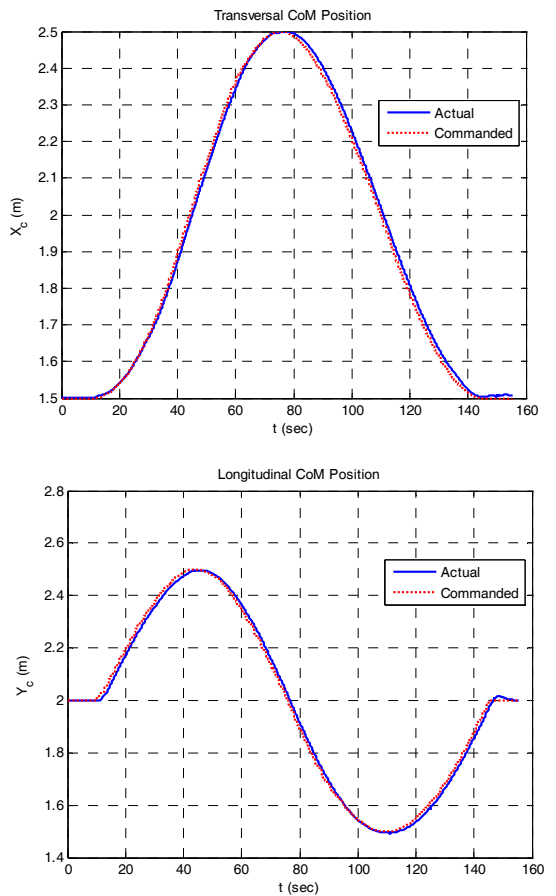

c)

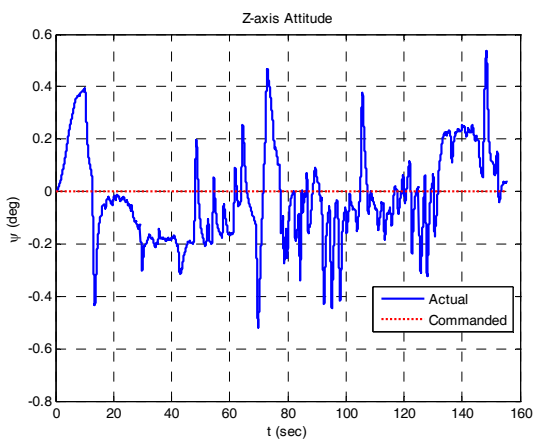

b)
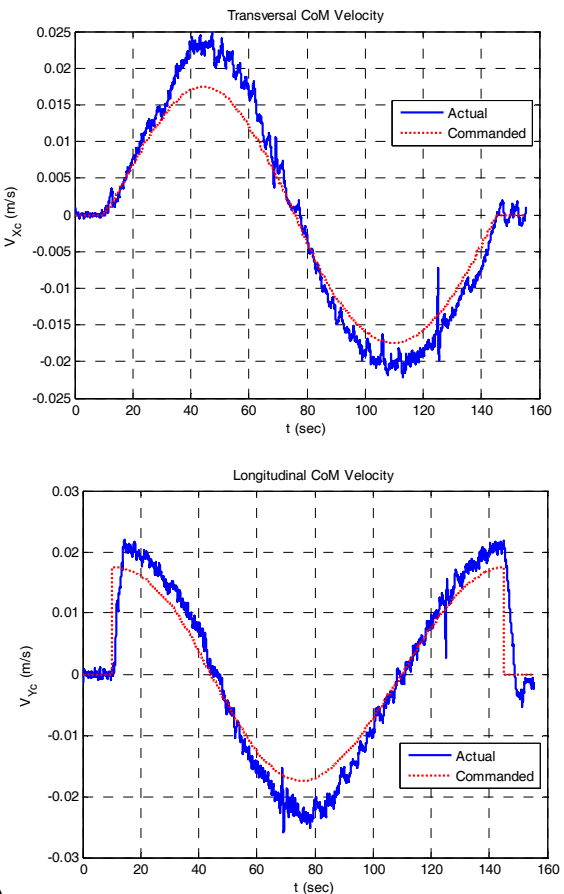

d)

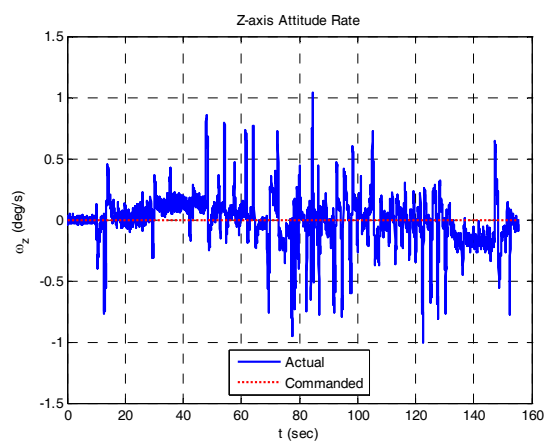

e)

Fig. 6. Logged data versus time of an autonomous proximity maneuver of NPS SRL's 3-DoF spacecraft simulator along a closed path using vectorable thrusters and MSGCMG. The simulator begins floating over the epoxy floor at $t=10 \mathrm{~s}$. a) Transversal position of the center of mass of the simulator in ICS; b) Transversal velocity of the center of mass of the simulator in ICS; c) Longitudinal position of the center of mass of the simulator; d) Longitudinal velocity of the center of mass of the simulator; e) Attitude; f) Attitude rate 
a)
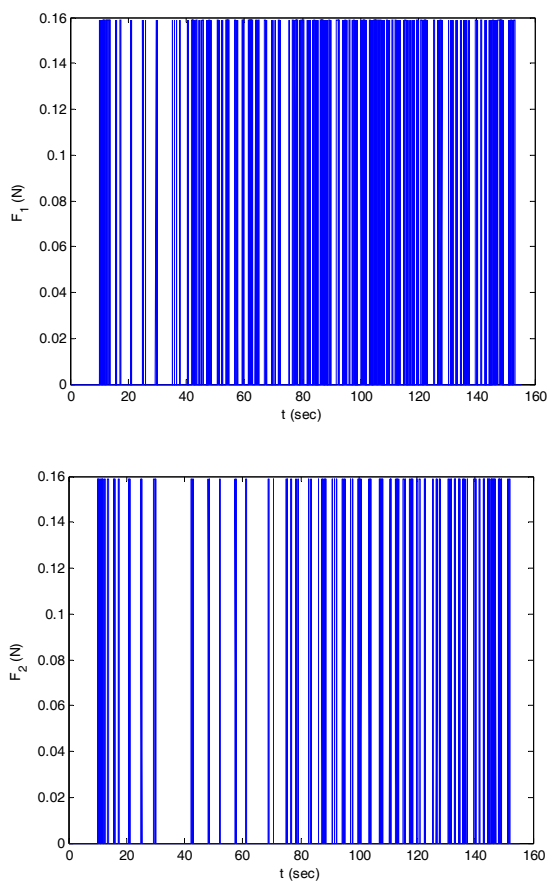

c)

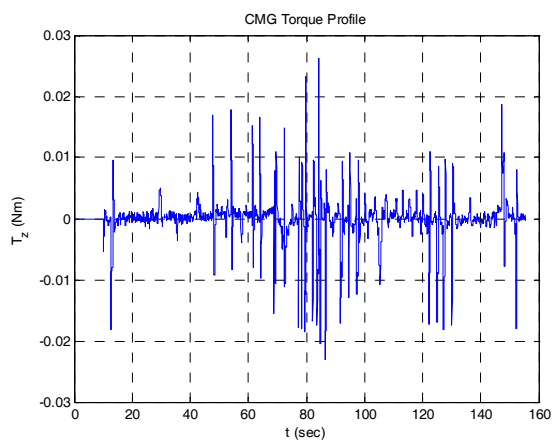

e)

d)

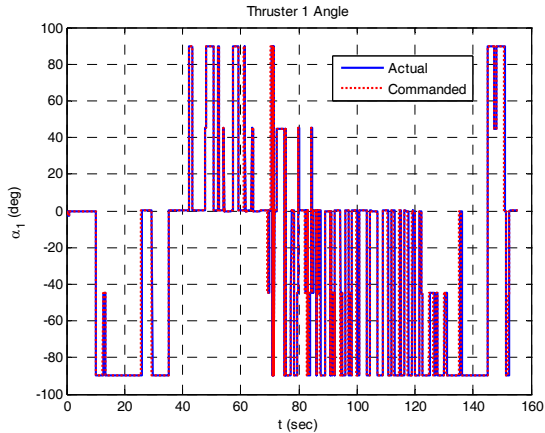

b)
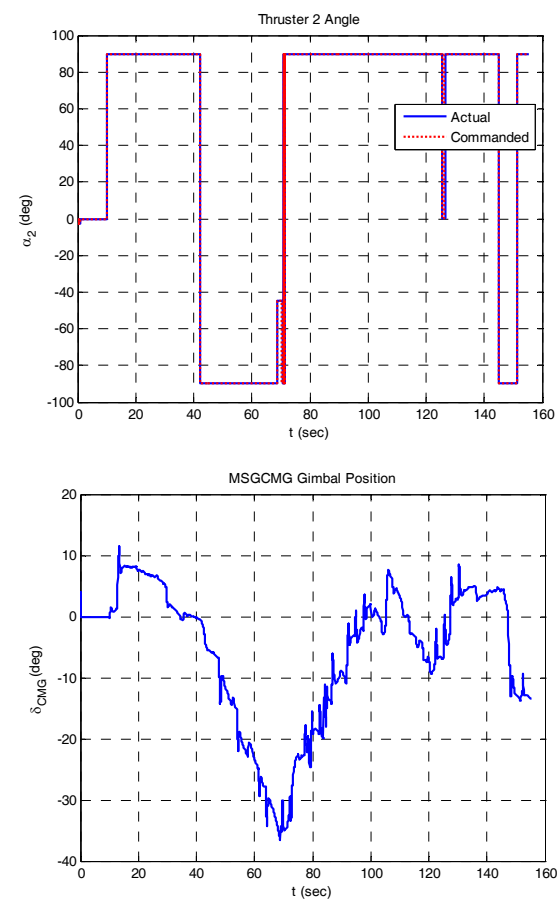

Fig. 7. Control actuator actions during autonomous proximity manuever of NPS SRL's 3DoF spacecraft simulator along a closed path using vectorable thrusters and MSGCMG. a) Thruster 1 firing profile; b) Thruster 1 position; c) Thruster 2 firing profile; d) Thruster 2 position; f) MSGCMG torque profile; e) MSGCMG gimbal position

Fig. 8 depicts a bird's-eye view of the spacecraft simulator motion. Of particular note, the good control accuracy can be evaluated by the closeness of the actual ground-track line to the commanded circular trajectory and of the initial configuration of the simulator to the final one. The total $\Delta V$ required during this experimental test was $.294 \mathrm{~m} / \mathrm{s}$ which correspond to a total impulse of $7.65 \mathrm{Ns}$. 


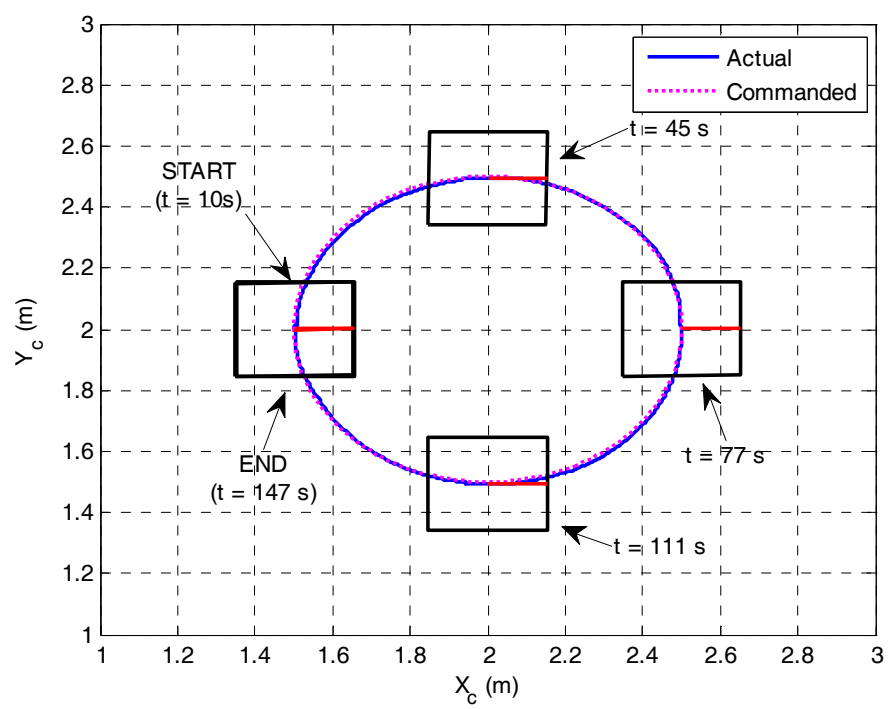

Fig. 8. Bird's-eye view of autonomous proximity manuever of NPS SRL's 3-DoF spacecraft simulator along a closed path using vectorable thrusters and MSGCMG

\subsection{Autonomous Proximity Maneuver using only Vectorable Thrusters along a Closed Circular Path}

Fig. 9, Fig. 10, and Fig. 11 report the results of maneuvering the spacecraft simulator along the same reference maneuver as in Section 6.1 but by using only the vectorable thrusters. This maneuver is presented to demonstrate the experimental validation of the STLC analytical results. As before, during the first $10 \mathrm{~s}$, the simulator is not floating and kept stationary while the attitude Kalman filter converges.

The tracking and estimation errors for this maneuver are as follows with the logged positions, attitudes and velocities shown in Fig. 9. The mean of the absolute value of the tracking error is $1.4 \mathrm{~cm}$ for $\Delta X$, with a standard deviation of $8.5 \mathrm{~mm}, 1.4 \mathrm{~cm}$ mean for $\Delta Y$ with a standard deviation of $8.6 \mathrm{~mm}, 2.5 \mathrm{~mm} / \mathrm{s}$ mean for $\Delta V_{X}$ with a standard deviation of $1.9 \mathrm{~mm} / \mathrm{s}$ and $3.1 \mathrm{~mm} / \mathrm{s}$ mean for $\Delta V_{Y}$ with a standard deviation of $2.8 \mathrm{~mm} / \mathrm{s}$. The mean of the absolute value of the estimated error in $X$ is $3 \mathrm{~mm}$ with a standard deviation of $3 \mathrm{~mm}$ and $4 \mathrm{~mm}$ in $Y$ with a standard deviation of $5 \mathrm{~mm}$. The mean of the absolute value of tracking error for $\Delta \psi$ is $0.52 \mathrm{deg}$ with a standard deviation of $0.31 \mathrm{deg}$ and $0.24 \mathrm{deg} / \mathrm{s}$ for $\Delta \omega_{z}$ with a standard deviation of $0.20 \mathrm{deg} / \mathrm{s}$. These control accuracies are in good agreement with the set parameters of the Schmitt triggers and LQR design.

Fig. 10 reports the command signals to the simulator's thrusters with the commands to the thrusters again demonstrating that the feedback linearized controller is able to determine the requisite thruster angles to take advantage of this fully minimized actuation system. Fig. 11 depicts a bird's-eye view of the motion of the simulator during this maneuver. The total $\Delta V$ required during this experimental test was $.327 \mathrm{~m} / \mathrm{s}$ which correspond to a total impulse of 8.55 Ns. 
a)
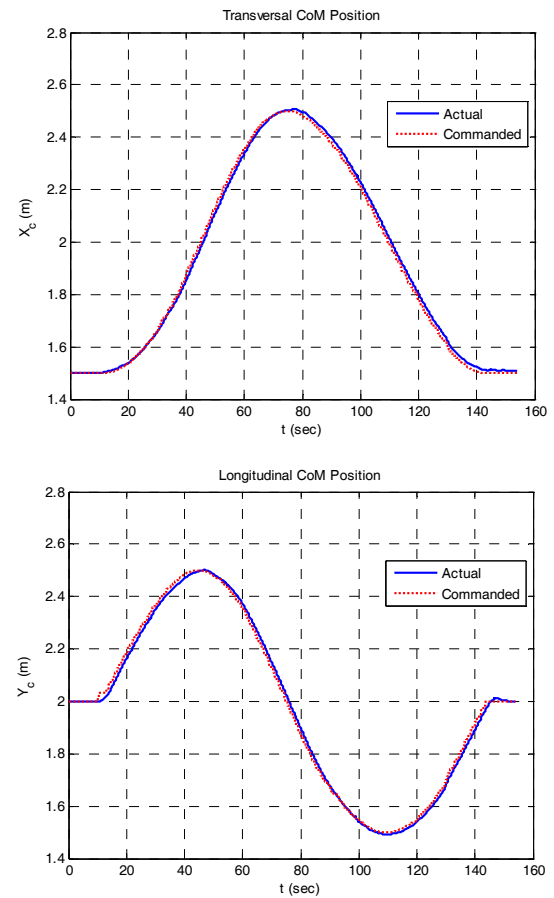

c)

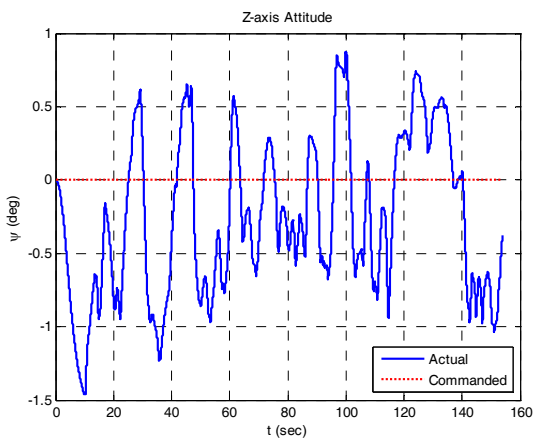

b)
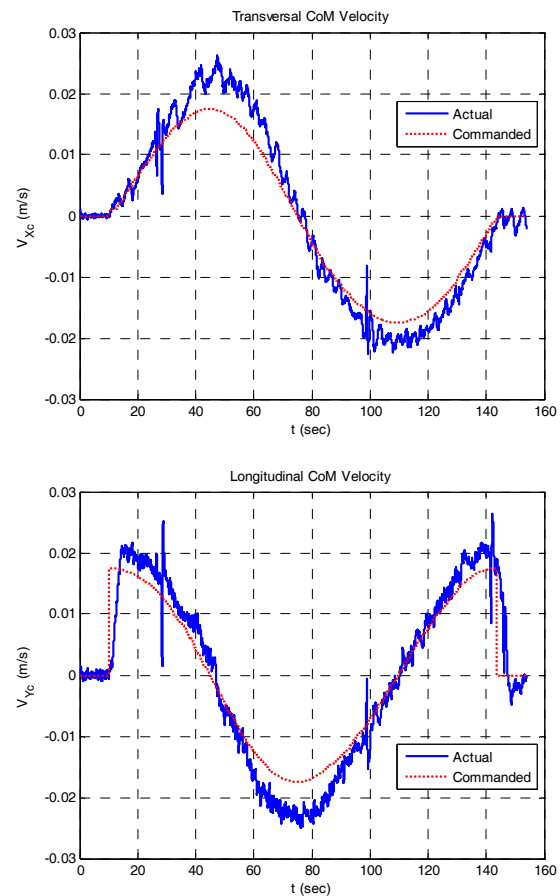

d)

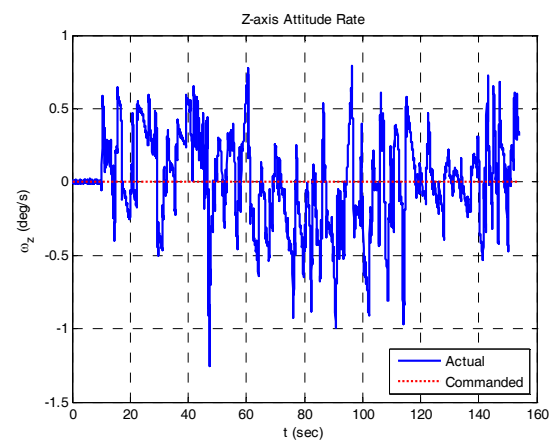

f)

e)

Fig. 9. Logged data versus time of an autonomous proximity maneuver of NPS SRL's 3-DoF spacecraft simulator along a closed path using only vectorable thrusters. The simulator begins floating over the epoxy floor at $\mathrm{t}=10 \mathrm{~s}$. a) Transversal position of the center of mass of the simulator in ICS; b) Transversal velocity of the center of mass of the simulator in ICS; c) Longitudinal position of the center of mass of the simulator; d) Longitudinal velocity of the center of mass of the simulator; e) Attitude; f) Attitude rate 
a)
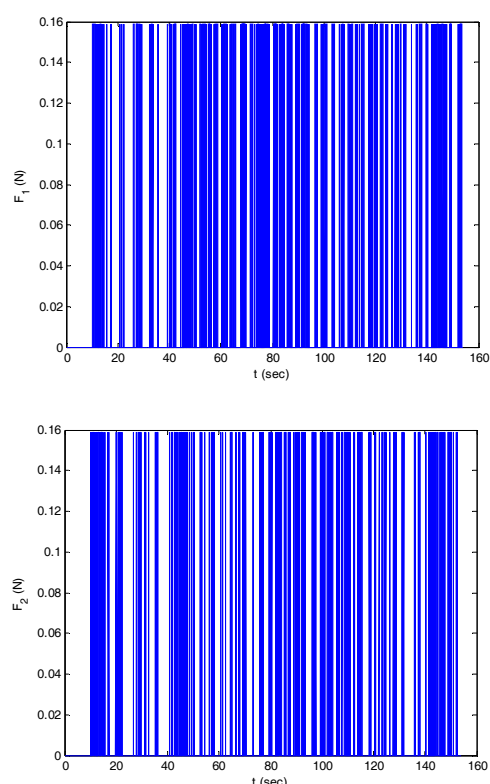

c)

Fig. 10. Control actuator actions during autonomous proximity manuever of NPS SRL's 3DoF spacecraft simulator along a closed path using only vectorable thrusters. a) Thruster 1 firing profile; b) Thruster 1 position; c) Thruster 2 firing profile; d) Thruster 2 position

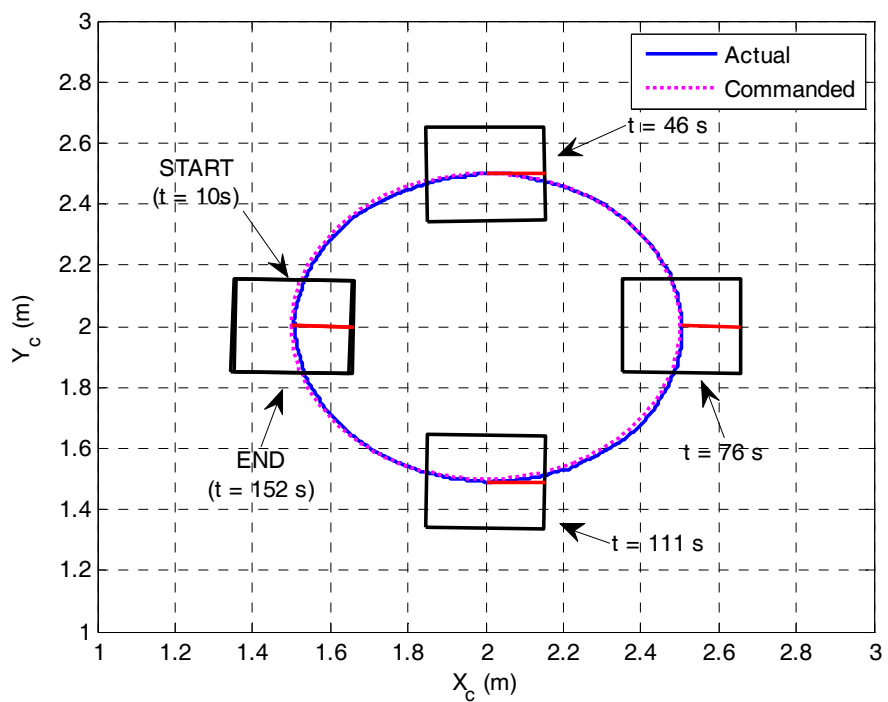

Fig. 11. Autonomous proximity maneuver of NPS SRL's 3-DoF spacecraft simulator along a closed path using only thrusters b)
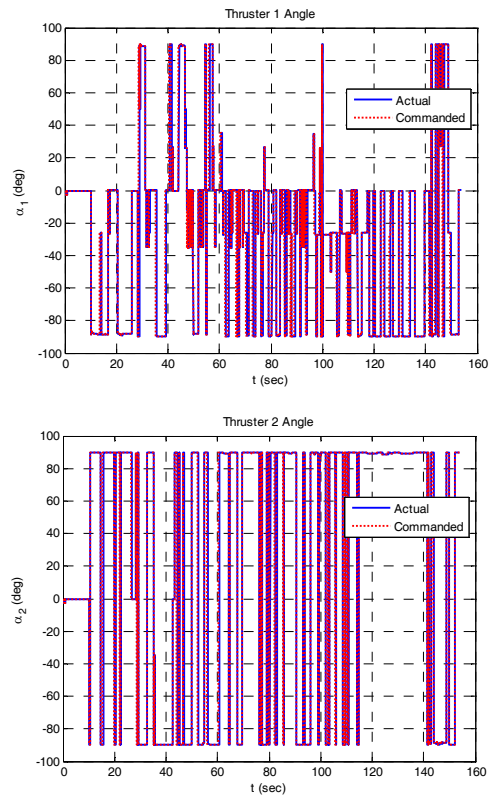


\section{Conclusion}

A planar laboratory testbed was introduced for the simulation of autonomous proximity maneuvers of a uniquely control actuator configured spacecraft. The testbed consists of a floating robotic simulator equipped with dual vectorable cold-gas thrusters and a miniature control moment gyro floating via planar air bearings on a flat floor. Inertial position and attitude measurements are obtained with a discrete Kalman filter and linear quadratic estimator for navigation; feedback linearized control coupled with a linear quadratic regulator is used to command the control moment gyro and while the same feedback linearized controller is used coupled with Schmitt triggers and Pulse Width Modulation to command the vectorable thrusters.

The presented experimental tests of autonomous closed path proximity maneuvers of the spacecraft simulator offer significant sample cases. The experimental results, which show good repeatability and robustness against disturbance and sensor noise, validate the proposed estimation and control approaches and demonstrate in particular, the small time local controllability of the system, confirming the analytical results. The achieved accuracy in following the reference trajectory (respectively $\sim 1 \mathrm{~cm}$ for translation and $\sim .5 \mathrm{deg}$ for rotation given only the vectorable thrusters as control inputs) demonstrates both a feasible and promising actuator configuration for small spacecraft.

NPS SRL's robotic spacecraft simulator testbed, despite its reduction to only 3-DoF, allows experiments to be conducted in a low-risk and relatively low-cost environment where intermediate validation can occur between analytical/numerical simulations and full flight proximity navigation missions. Furthermore, the controllability analysis and the algorithms proposed for the state estimation and control can be in principle extended to full-fidelity 6DoF spacecraft applications. The next step in this ongoing research will focus on the expansion of the presented analytical methods for non-linear control-affine systems with drift to numerical simulations on a full 6-DoF spacecraft model as well as work to develop further controllers that can take advantage of the minimum number of control actuator configuration of only two thrusters and no momentum exchange devices.

\section{References}

Bullo, F. \& Lewis, A.D. (2005). Geometric Control of Mechanical Systems, Springer Science+Business Media, Inc., ISBN:0-387-22195-6, New York, NY, USA

Bryson, A.E. (1994). Control of Spacecraft and Aircraft, Princeton University Press, ISBN: 0-69108782-2, Princeton, NJ, USA

Bevilacqua, R., Hall, J.S., Horning, J. \& Romano, M. (2009). Ad Hoc Networking and Shared Computation Based Upon Linux for Autonomous Multi-Robot Systems, Journal of Aerospace Computing, Information, and Communication. To Appear.

Canfield, S.L. \& Reinholtz, C.F. (1998). Development of the Carpal Robotic Wrist, Lecture Notes in Control and Information Sciences, Vol. 232, pp. 423-434, ISBN: 978-3-54076218-8, Springer Berlin

Corrazzini, T. \& How, J.P. (1998) Onboard GPS Signal Augmentation for Spacecraft Formation Flying, Proceedings of the $11^{\text {th }}$ International Technical Meeting of the Satellite Division of the Institute of Naviagation (ION) GPS 1998, pp. 1937-1946, Nashville, TN, September 1998, ION, Manassas, VA, USA 
Crassidis, J.L. \& Junkins, J.L. (2004). Optimal Estimation of Dynamic Systems, CRC Press, LLC, ISBN: 1-58488-391-X, Boca Raton, FL, USA

Creamer, G. (2007). The SUMO/FREND Project: Technology Development for Autonomous Grapple of Geosynchronous Satellites, Advances in the Astronautical Sciences, Vol. 128, pp. 895-910, ISBN: 978-0-87703-542-8, San Diego, CA, USA

Eikenberry, B.D. (2006). Guidance and Navigation Software Architecture Design for the Autonomous Multi-Agent Physically Interacting Spacecraft (AMPHIS) Testbed, M.S. Thesis, Naval Postgraduate School, Monterey, CA, USA

Gelb, A. (1974). Applied Optimal Estimation, The MIT Press, ISBN: 0-262-57048-3, Cambridge, MA, USA

Hall, J.S. (2006). Design and Interaction of a Three Degrees-of-Freedom Robotic Vehicle with Control Moment Gyro for the Autonomous Multi-Agent Physically Interacting Spacecraft (AMPHIS) Testbed, M.S. Thesis, Naval Postgraduate School, Monterey, CA, USA

Hall, J.S. \& Romano, M. (2007). Autonomous Proximity Operations of Small Satellites with Minimum Numbers of Actuators, Proceedings of the 21st AIAA/USU Small Satellite Conference, Logan, UT, USA, August 2007, AIAA/USU

Hall, J.S. \& Romano, M. (2007). Novel Robotic Spacecraft Simulator with Mini-Control Moment Gyroscopes and Rotating Thrusters, Proceedings of the 2007 IEEE/ASME International Conference on Advanced Intelligent Mechatronics, pp. 1-6, ISBN: 878-14244-1264-8, Zurich, Switzerland, September 2007, IEEE

Isidori, A. (1989). Nonlinear Control Systems: An Introduction, Springer-Verlag New York, Inc., ISBN: 0-387-50601-2, New York, NY, USA

Kennedy, F. (2008). Orbital Express: Accomplishments and Lessons Learned, Advances in the Astronautical Sciences, Vol. 131, pp. 575-586, ISBN: 878-0-87703-545-9, San Diego, CA, USA

Lappas, V.J.; Steyn, W.H. \& Underwood, C.I. (2002). Practical Results on the Development of a Control Moment Gyro Based Attitude Control System for Agile Small Satellites, Proceedings of the $16^{\text {th }}$ Annual AIAA/USU Small Satellite Conference, Logan, UT, USA, August 2002, AIAA/USU

LaValle, S.M. (2006). Planning Algorithms, Cambridge University Press, ISBN: 0-521-86205-1, New York, NY, USA

Ledebuhr, A.G.; Ng, L.C.; Jones, M.S.; Wilson, B.A.; Gaughan, R.J.; Breitfeller, E.F.; Taylor, W.G.; Robinson, J.A.; Antelman, D.R. \& Nielsen, D.P. (2001). Micro-Satellite Ground Test Vehicle for Proximity and Docking Operations Development, Proceedings of the 2001 Aerospace Conference, Vol. 5, pp. 2493-2504, ISBN: 0-7803-65992, Big Sky, MT, USA, March 2001, IEEE

LeMaster, E.A; Schaechter, D.B \& Carrington, C.K. (2006). Experimental Demonstration of Technologies for Autonomous On-Orbit Robotic Assembly, Space 2006, pp. 1-13, San Jose, CA, USA, September 2006, AIAA

Lewis, A.D. \& Murray, R.M. (1997). Configuration Controllability of Simple Mechanical Control Systems, SIAM Journal on Control and Optimization, Vol. 35, No. 3, pp. 766790, SIAM

Lugini, C. \& Romano, M. (2009). A ballistic-pendulum test stand to characterize small coldgas thruster nozzles, Acta Astronautica, Vol. 64, No. 5-6, pp. 615-625, Elsevier LTD, DOI: $10.1016 /$ j.actaastro.2008.11.001 
Machida, K.; Toda, Y. \& Iwata, T. (1992). Maneuvering and Manipulation of Flying Space Telerobotics System, Proceedings of the 1992 IEEE/RSJ International Conference on Intelligent Robots and Systems, Vol. 1, pp. 3-10, ISBN: 0-7803-0737-2, Raleigh, NC, USA, July 1992, IEEE

Marchesi, M.; Angrilli, F. \& Venezia, R. (2000). Coordinated Control for Free-flyer Space Robots, Proceedings of the 2000 IEEE International Conference on Systems, Man, and Cybernetics, Vol. 5, pp. 3550-3555, ISBN: 0-7803-6583-6, Nashville, TN, USA, October 2000, IEEE

Mathieu, C. \& Weigel, A. L. (2005). Assessing the Flexibility Provided by Fractionated Spacecraft, Space 2005, pp. 1-12, Long Beach, CA, USA, August 2005, AIAA

Nolet, S.; Kong, E. \& Miller, D.W. (2005). Design of an Algorithm for Autonomous Docking with a Freely Tumbling Target, Proceedings of Modeling, Simulation and Verification of Space-based Systems II, Vol. 5799, No. 123, pp. 123-134, Orlando, FL, USA, March 2005, SPIE

Price, W. (2006). Control System of a Three DOF Spacecraft Simulator by Vectorable Thrusters and Control Moment Gyros, M.S. Thesis, Naval Postgraduate School, Monterey, CA, USA

Romano, M.; Friedman, A. \& Shay, T.J. (2007). Laboratory Experimentation of Autonomous Spacecraft Approach and Docking to a Collaborative Target, Journal of Spacecraft and Rockets, Vol. 44, No. 1, pp. 164-173, DOI: 10.2514/1.22092, AIAA

Romano, M. \& Hall, J.S (2006). A Testbed for Proximity Navigation and Control of Spacecraft for On-orbit Assembly and Reconfiguration, Space 2006, pp. 1-11, San Jose, CA, USA, September 2006, AIAA

Roser, X. \& Sghedoni, M. (1997). Control Moment Gyroscopes (CMG's) and their Applications in Future Scientific Missions, Proceedings of the $3^{\text {rd }}$ ESA International Conference on Spacecraft Guidance, Navigation and Control Systems, pp. 523-528, ESTEC Noordwijk, the Netherlands, November 1996, European Space Agency

Sussman, H.J. (1987). A General Theorem on Local Controllability, SIAM Journal on Control and Optimization, Vol. 25, No. 1, pp. 158-194, SIAM

Sussman, H.J. (1990). Nonlinear Controllability and Optimal Control, Marcel Dekker, Inc., ISBN: 0-8247-8258-5, New York, NY, USA

Slotine, J.E. \& Weiping, L. (1991). Applied Nonlinear Control, Prentice-Hall, Inc., ISBN: 0-13040890-5, Upper Saddle River, NJ, USA

Ullman, M.A. (1993). Experimentation in Autonomous Navigation and Control of MultiManipulator Free-Flying Space Robots, Ph.D. Dissertation, Stanford University, Stanford, CA, USA

Wie, B. (1998). Space Vehicle Dynamics and Control, American Institute of Aeronautics and A stronautics, Inc., ISBN: 1-56347-261-9, Reston, VA, USA 


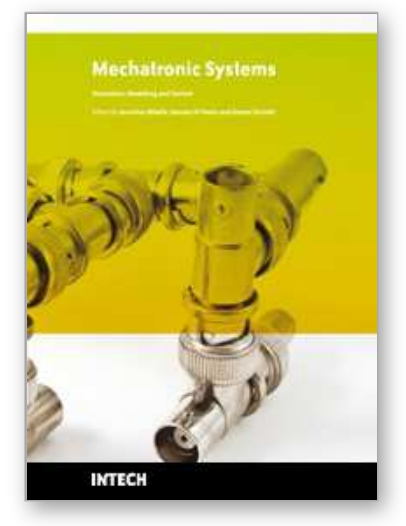

\author{
Mechatronic Systems Simulation Modeling and Control \\ Edited by Annalisa Milella Donato Di Paola and Grazia Cicirelli
}

ISBN 978-953-307-041-4

Hard cover, 298 pages

Publisher InTech

Published online 01, March, 2010

Published in print edition March, 2010

This book collects fifteen relevant papers in the field of mechatronic systems. Mechatronics, the synergistic blend of mechanics, electronics, and computer science, integrates the best design practices with the most advanced technologies to realize high-quality products, guaranteeing at the same time a substantial reduction in development time and cost. Topics covered in this book include simulation, modelling and control of electromechanical machines, machine components, and mechatronic vehicles. New software tools, integrated development environments, and systematic design methods are also introduced. The editors are extremely grateful to all the authors for their valuable contributions. The book begins with eight chapters related to modelling and control of electromechanical machines and machine components. Chapter 9 presents a nonlinear model for the control of a three-DOF helicopter. A helicopter model and a control method of the model are also presented and validated experimentally in Chapter 10. Chapter 11 introduces a planar laboratory testbed for the simulation of autonomous proximity manoeuvres of a uniquely control actuator configured spacecraft. Integrated methods of simulation and Real-Time control aiming at improving the efficiency of an iterative design process of control systems are presented in Chapter 12. Reliability analysis methods for an embedded Open Source Software (OSS) are discussed in Chapter 13. A new specification technique for the conceptual design of self-optimizing mechatronic systems is presented in Chapter 14 . Chapter 15 provides a general overview of design specificities including mechanical and control considerations for micro-mechatronic structures. It also presents an example of a new optimal synthesis method to design topology and associated robust control methodologies for monolithic compliant microstructures.

\title{
How to reference
}

In order to correctly reference this scholarly work, feel free to copy and paste the following:

Jason S. Hall and Marcello Romano (2010). Laboratory Experimentation of Guidance and Control of Spacecraft During On-Orbit Proximity Maneuvers, Mechatronic Systems Simulation Modeling and Control, Annalisa Milella Donato Di Paola and Grazia Cicirelli (Ed.), ISBN: 978-953-307-041-4, InTech, Available from: http://www.intechopen.com/books/mechatronic-systems-simulation-modeling-and-control/laboratoryexperimentation-of-guidance-and-control-of-spacecraft-during-on-orbit-proximity-maneuvers

\section{INTECH}

open science | open minds

\author{
InTech Europe \\ University Campus STeP Ri
}

\section{InTech China}

Unit 405, Office Block, Hotel Equatorial Shanghai 
Slavka Krautzeka 83/A

51000 Rijeka, Croatia

Phone: +385 (51) 770447

Fax: +385 (51) 686166

www.intechopen.com
No.65, Yan An Road (West), Shanghai, 200040, China 中国上海市延安西路65号上海国际贵都大饭店办公楼405单元 Phone: +86-21-62489820

Fax: $+86-21-62489821$ 
(C) 2010 The Author(s). Licensee IntechOpen. This chapter is distributed under the terms of the Creative Commons Attribution-NonCommercialShareAlike-3.0 License, which permits use, distribution and reproduction for non-commercial purposes, provided the original is properly cited and derivative works building on this content are distributed under the same license. 